\title{
143.
}

\section{TABLES OF THE COVARIANTS M TO W OF THE BINARY QUIN- TIC: FROM THE SECOND, THIRD, FIFTH, EIGHTH, NINTH AND TENTH MEMOIRS ON QUANTICS.}

[Arranged in the present form, 1889.]

THE binary quintic has in all (including the quintic itself and the invariants) 23 covariants, which I have represented by the capital letters, A, B, C, ...W (alternative forms of two of these are denoted by $Q^{\prime}$ and $S^{\prime}$ ). The covariants $A, \ldots L$, and also $Q, Q^{\prime}$ were given in my Second Memoir on Quantics, and except $Q$ and $Q^{\prime}$ are reproduced in the present reprint thereof, $141 ;$ in all these I gave not only the literal terms actually presenting themselves, but also the terms with zero coefficients; in the other covariants however, or in most of them, the terms with zero coefficients were omitted. It is very desirable to have in every case the complete series of literal terms, and in the covariants as here printed they are accordingly inserted: the number of terms is in each case known beforehand by the foregoing af-table, 142, and any omission is thus precluded; by means of this af-table we have the numbers of terms as shown in the following list.

I have throughout (as was done in the Ninth and Tenth Memoirs) expressed the literal terms in a slightly different form from that employed in the Second Memoir: this is done in order to show at a glance in each column the set of terms which contain a given power of $a$, and in each such set the terms which contain a given power of $b$.

The numerical verifications are also given not only for the entire column but for each set of terms containing the same power of $a$; viz. in most cases, but not always, the positive and negative coefficients of a set have equal sums, which are shown by 
a number with the sign \pm prefixed. The verification is in some cases given in regard to the subsets involving the same powers of $a$ and $b$, here also the sums of the positive and negative coefficients are not in every case equal. The cases of inequality will be referred to at the end of this paper.

The whole series of covariants is as follows:

Mem. No. of table.

deg-weight

\begin{tabular}{|c|c|c|c|c|}
\hline 2 & 13 & A & $\left(1,1,1,1,1,1 \gamma(x, y)^{5}\right.$ & $1(0 \ldots 5)$ \\
\hline " & 14 & B & $\left(3,3,3 \gamma(x, y)^{2}\right.$ & $2(4.6)$ \\
\hline " & 15 & $\mathrm{C}$ & $\left(2,2,3,3,3,2,2 \gamma(x, y)^{6}\right.$ & $2(2 \ldots \ldots 8)$ \\
\hline$"$ & 16 & $\mathrm{D}$ & $\left(6,6,6,6 \gamma(x, y)^{3}\right.$ & $3(6 \ldots 9)$ \\
\hline$"$ & 17 & $\mathrm{E}$ & $\left(5,6,6,6,6,5 \gamma(x, y)^{5}\right.$ & $3(5 \ldots 10)$ \\
\hline$”$ & 18 & $\mathrm{~F}$ & $=\left(3,4,5,6,6,6,6,5,4,3 \gamma(x, y)^{9}\right.$ & $3(3 \ldots$ \\
\hline , & 19 & G & $\left(12 \gamma(x, y)^{0}\right.$, Invt. & $4-10$ \\
\hline$"$ & 20 & $\mathrm{H}$ & $\left(11,11,12,11,11 \gamma(x, y)^{4}\right.$ & $4(8 \ldots 12)$ \\
\hline$"$ & 21 & I & $=\left(9,11,11,12,11,11,9 \gamma(x, y)^{6}\right.$ & $4(7 \ldots \ldots 13)$ \\
\hline$”$ & 22 & $\mathbf{J}$ & $\left(20,20 \gamma(x, y)^{1}\right.$ & $5(12,13)$ \\
\hline " & 23 & $\mathrm{~K}$ & $\left(19,20,20,19 \gamma(x, y)^{3}\right.$ & $5(11 \ldots 14)$ \\
\hline ", & 24 & $\mathrm{~L}$ & $=\left(16,18,19,20,20,19,18,16 \gamma(x, y)^{7}\right.$ & $5(9 \ldots \ldots 16)$ \\
\hline 8 & 83 & M & $(32,32,32 \gamma x, y)^{2}$ & $6(14.16)$ \\
\hline$"$ & 84 & $\mathrm{~N}$ & $\left(30,32,32,32,30 \gamma(x, y)^{4}\right.$ & $6(13 \ldots 17)$ \\
\hline 9 & 90 & 0 & $\left(49,49 \gamma(x, y)^{1}\right.$ & $7(16,18)$ \\
\hline$"$ & 91 & $\mathrm{P}$ & $=\left(46,48,49,49,48,46 \gamma(x, y)^{5}\right.$ & $7(15 \ldots 20)$ \\
\hline 2 & $\begin{array}{l}\text { Q } 25 \\
Q^{\prime} 26\end{array}$ & $\mathrm{Q}, \mathrm{Q}^{\prime}$ & $\left(73 \gamma(x, y)^{0}\right.$, Invt. & $8-20$ \\
\hline 9 & 92 & $\mathrm{R}$ & $\left(71,73,71 \gamma(x, y)^{2}\right.$ & $8(19.21)$ \\
\hline $1 \theta$ & $\begin{array}{l}\text { S } 93 \\
\text { S } 93 \text { bis }\end{array}$ & $\mathrm{S}, \mathrm{S}^{\prime}$ & $\left(101,102,102,1.01 \gamma(x, y)^{3}\right.$ & $9(21 \ldots 24)$ \\
\hline 9 & 94 & $\mathrm{~T}$ & $\left(190,190 \gamma(x, y)^{1}\right.$ & $11(27,28)$ \\
\hline 3 & 29 & $\mathrm{U}$ & $(252 \gamma x, y)^{0}$, Invt. & $12-30$ \\
\hline 9 & 95 & $\mathrm{~V}$ & $\left(325,325 \gamma(x, y)^{1}\right.$ & $13(32,33)$ \\
\hline 5 & $29 \mathrm{~A}$ & $\mathrm{~W}$ & $\left(967 \gamma(x, y)^{0}\right.$, Invt. & $18-45$ \\
\hline
\end{tabular}


M. No. 83 .

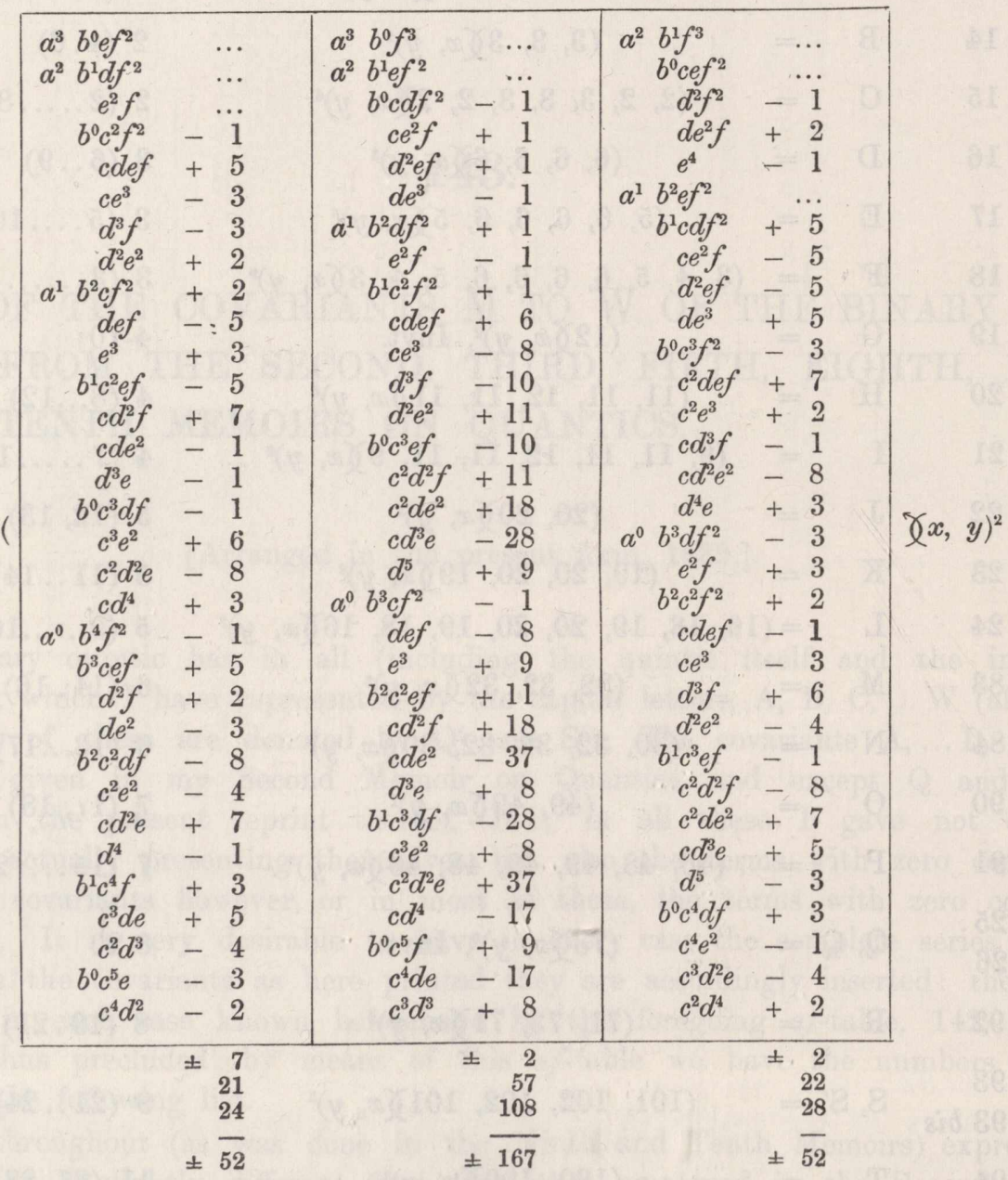


O. No. 90 .

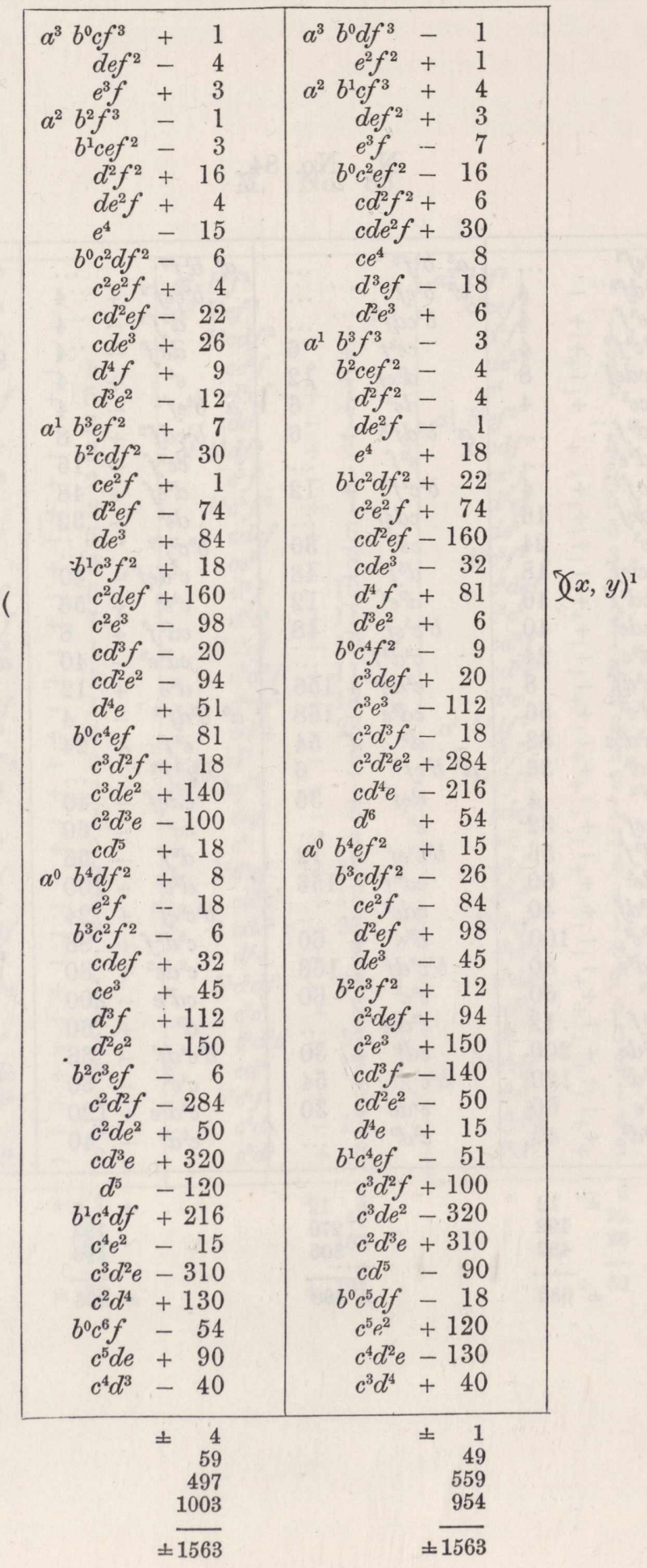


P. No. 91 .

\begin{tabular}{|c|c|c|c|c|c|c|c|}
\hline $\begin{array}{c}a^{4} b^{0} f^{3} \\
a^{3} b^{1} e f^{2} \\
b^{0} c d f^{2} \\
c e^{2} f \\
d^{2} e f \\
d e^{3} \\
a^{2} b^{2} d f^{2} \\
e^{2} f \\
b^{1} c^{2} f^{2} \\
c d e f \\
c e^{3} \\
d^{3} f \\
d^{2} e^{2} \\
b^{0} c^{3} e f \\
c^{2} d^{2} f \\
c^{2} d e^{2} \\
c d^{3} e \\
d^{5} \\
a^{1} b^{3} c f^{2} \\
d e f \\
e^{3} \\
b^{2} c^{2} e f \\
c d^{2} f \\
c d e^{2} \\
d^{3} e \\
b^{1} c^{3} d f \\
c^{3} e^{2} \\
c^{2} d^{2} e \\
c d^{4} \\
b^{0} c^{5} f \\
c^{4} d e \\
c^{3} d^{3} \\
a^{0} b^{5} f^{2} \\
b^{4} c e f \\
d^{2} f \\
d e^{2} \\
b^{3} c^{2} d f \\
c^{2} e^{2} \\
c d^{2} e \\
d^{4} \\
b^{2} c^{4} f \\
c^{3} d e \\
c^{2} d^{3} \\
b^{1} c^{5} e \\
c^{4} d^{2} \\
b^{0} c^{6} d\end{array}$ & 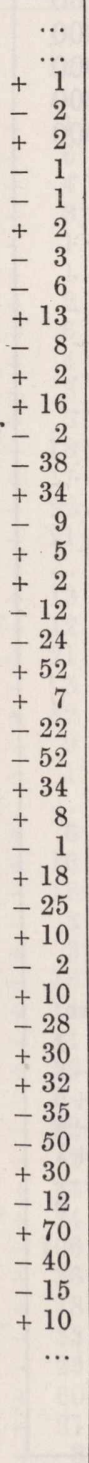 & $\begin{array}{c}a^{3} b^{2} f^{3} \\
b^{0} c e f^{2} \\
d^{2} f^{2} \\
d e^{2} f \\
e^{4} \\
a^{2} b^{2} e f^{2} \\
b^{1} c d f^{2} \\
c e^{2} f \\
d^{2} e f \\
d e^{3} \\
b^{0} c^{3} f^{2} \\
c^{2} d e f \\
c^{2} e^{3} \\
c d^{3} f \\
c d^{2} e^{2} \\
d^{4} e \\
a^{1} b^{3} d f^{2} \\
e^{2} f \\
b^{2} c^{2} f^{2} \\
c d e f \\
c e^{3} \\
d^{3} f \\
d^{2} e^{2} \\
b^{1} c^{3} e f \\
c^{2} d^{2} f \\
c^{2} d e^{2} \\
c d^{3} e \\
d^{5} \\
b^{0} c^{4} d f \\
c^{4} e^{2} \\
c^{3} d^{2} e \\
c^{2} d^{4} \\
a^{0} b^{4} c f^{2} \\
d e f \\
e^{3} \\
b^{3} c^{2} e f \\
c d^{2} f \\
c d e^{2} \\
d^{3} e \\
b^{2} c^{3} d f \\
c^{3} e^{2} \\
c^{2} d^{2} e \\
c d^{4} \\
b^{1} c^{5} f \\
c^{4} d e \\
c^{3} d^{3} \\
b^{0} c^{6} e \\
c^{5} d^{2} \\
\end{array}$ & $\begin{array}{lr} & \cdots \\
+\quad 5 \\
+\quad 1 \\
-\quad 2 \\
+\quad 2 \\
-\quad 17 \\
+\quad 13 \\
-\quad 32 \\
+\quad 32 \\
+\quad 4 \\
+\quad 36 \\
-\quad 24 \\
-\quad 10 \\
-\quad 16 \\
+\quad 12 \\
+\quad 7 \\
-\quad 12 \\
+\quad 6 \\
+\quad 42 \\
+\quad \ldots \\
+\quad 54 \\
-\quad 91 \\
-\quad 68 \\
-\quad 64 \\
+\quad 14 \\
+204 \\
-\quad 93 \\
+\quad 37 \\
+\quad 86 \\
-208 \\
+\quad 86 \\
-\quad 5 \\
-\quad 12 \\
+\quad 3 \\
+\quad 34 \\
+\quad 46 \\
+105 \\
-\quad 20 \\
+\quad 50 \\
-110 \\
-170 \\
+115 \\
-\quad 21 \\
+250 \\
-150 \\
-\quad 60 \\
+\quad 40\end{array}$ & 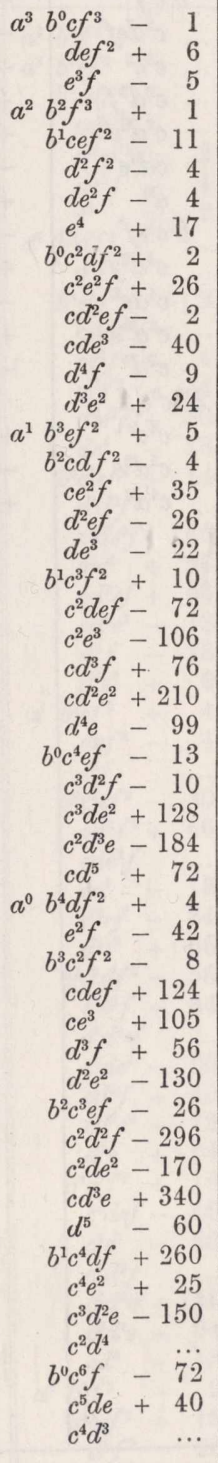 & 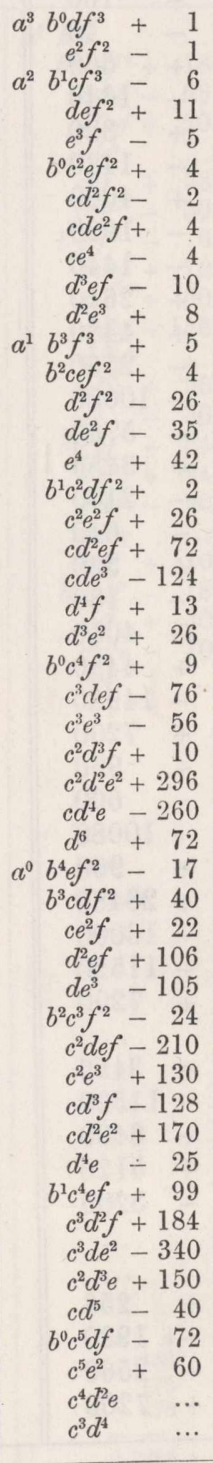 & 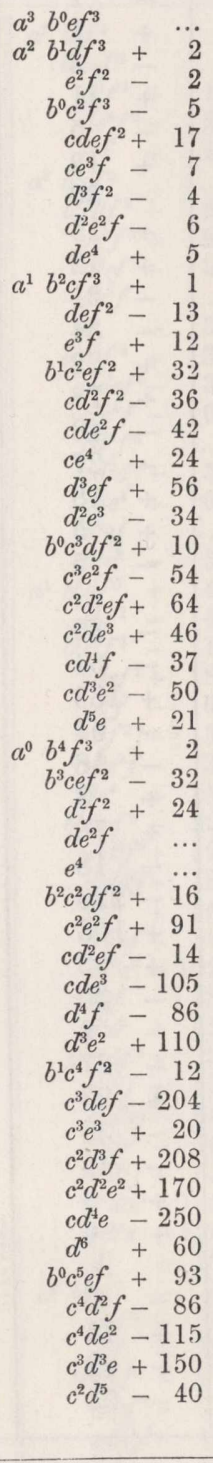 & 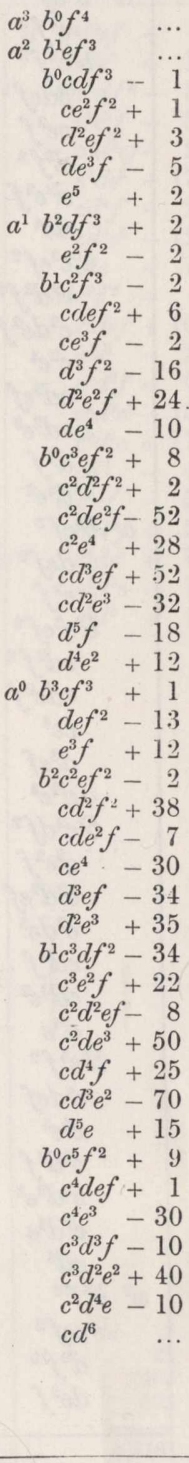 \\
\hline & $\begin{array}{rr} \pm \quad 3 \\
67 \\
136 \\
182\end{array}$ & & $\begin{array}{r}5 \\
\pm \quad 99 \\
536 \\
594\end{array}$ & $\begin{array}{r}6 \\
7 \quad 70 \\
536 \\
954\end{array}$ & $\begin{array}{r}1 \\
27 \\
577 \\
961 \\
\end{array}$ & $\begin{array}{r}244 \\
266 \\
944 \\
\end{array}$ & $\begin{array}{r} \pm \quad 6 \\
134 \\
248 \\
\end{array}$ \\
\hline & & & & \pm 1566 & 66 & & \\
\hline
\end{tabular}


Q. No. 25 . Q'. No. 26 .

Q. No. 25. Q'. No. 26 .

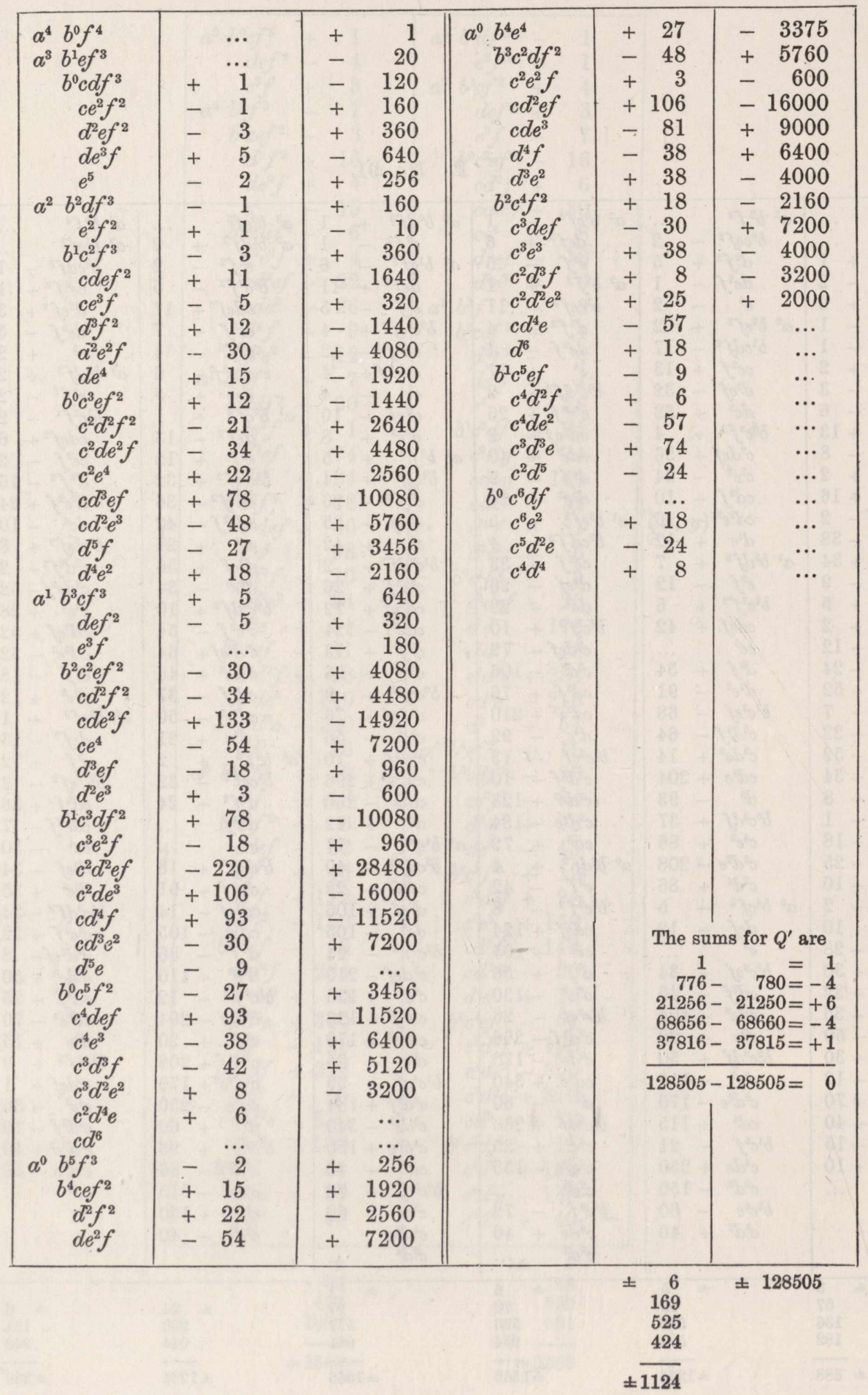


R. No. 92 .

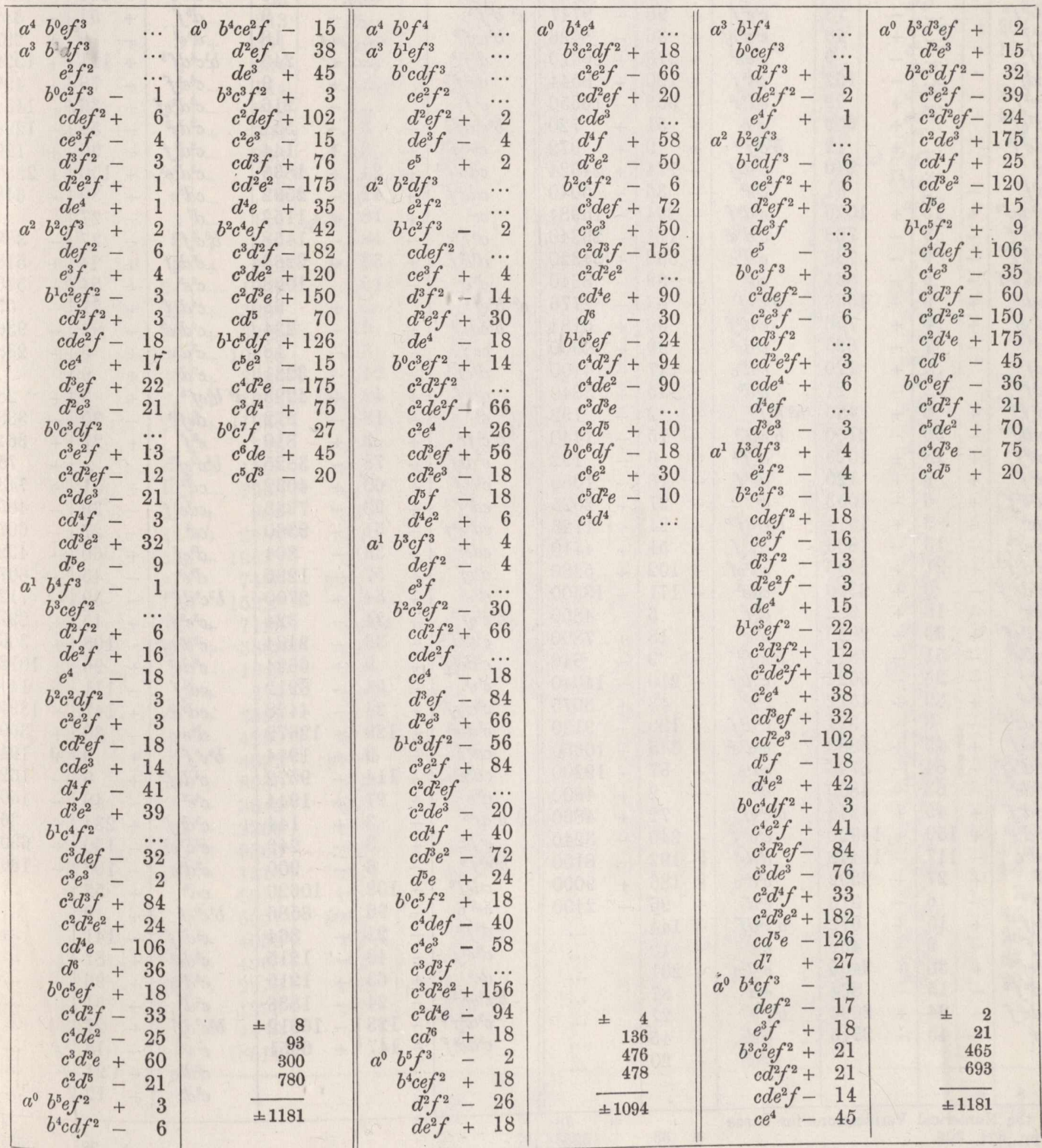

C. II. 
S. No. 93 bis; S'. No. 93 . $\left(*^{\Upsilon}(x, y)^{3}\right.$.

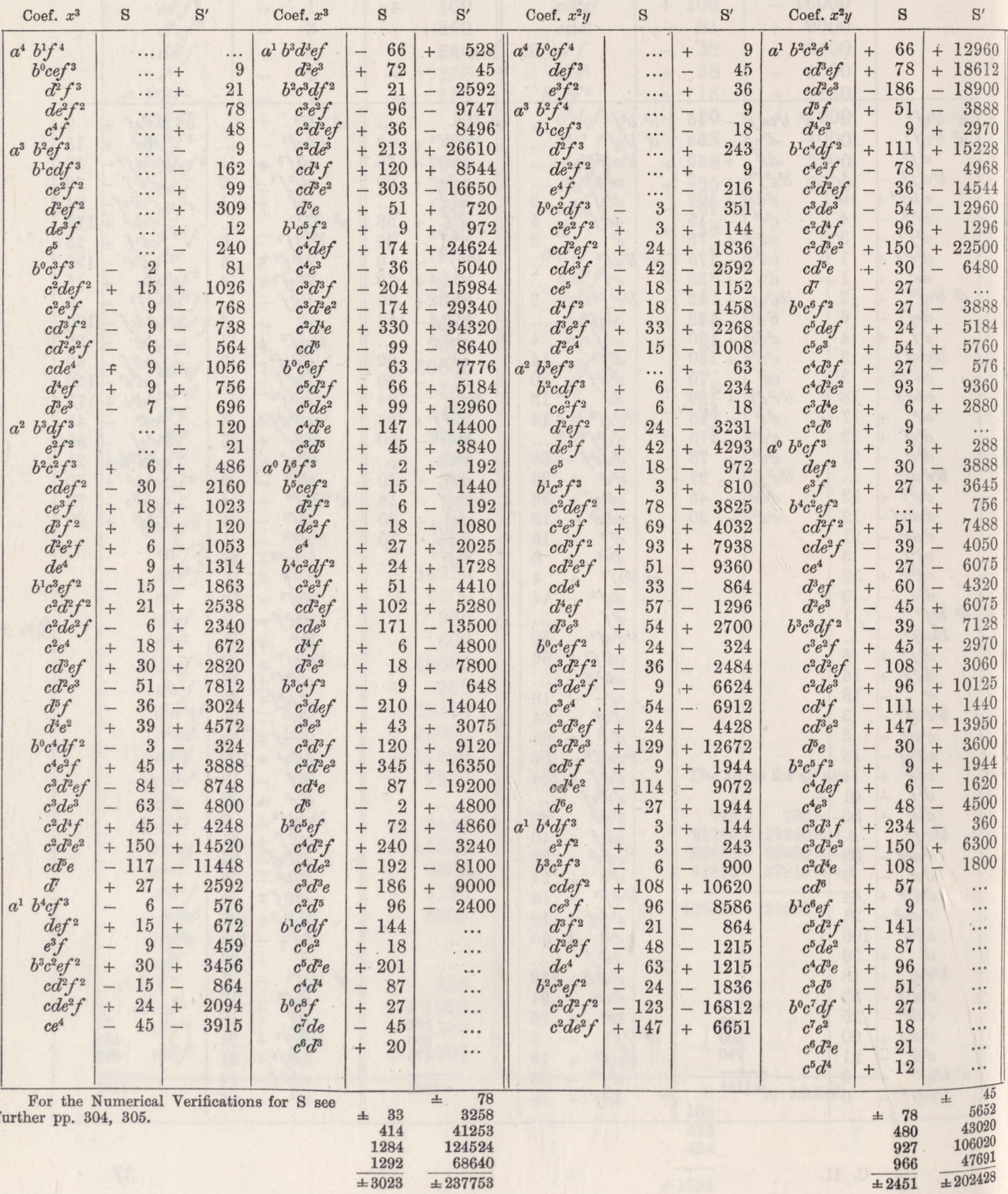


S. No. 93 bis; S'. No. 93.

\begin{tabular}{|c|c|c|c|c|c|c|c|c|c|c|c|}
\hline Coef. $x y^{2}$ & $\mathrm{~S}$ & $\mathbf{S}^{\prime}$ & Coef. x & S & $\mathbf{S}^{\prime}$ & ef. $y^{3}$ & $\mathrm{~S}$ & $\mathbf{S}^{\prime}$ & oef. $y^{3}$ & 8 & \\
\hline $\begin{array}{c}a^{4} b^{0} d f^{4} \\
e^{2} f^{3} \\
a^{3} b^{1} c f^{4} \\
d e f^{3} \\
e^{3} f^{2} \\
b^{0} c^{2} e f^{3} \\
c d^{2} f^{3} \\
c d e^{2} f^{2} \\
c e^{4} f \\
d^{3} e f^{2} \\
d^{2} e^{3} f \\
d e^{5} \\
x^{2} b^{3} f^{4} \\
b^{2} c e f^{3} \\
d^{2} f^{3} \\
d^{2} f^{2} \\
e^{4} f^{4} \\
b^{1} c^{2} d f^{3} \\
c^{2} e^{2} f^{2} \\
c d^{2} e f^{2} \\
c d e^{3} f \\
c e^{5} \\
d^{4} f^{2} \\
d^{3} e^{2} f \\
d^{2} e^{4} \\
b^{0} c^{4} f^{3} \\
c^{3} d e f^{2} \\
c^{3} e^{3} f \\
c^{2} d^{3} f^{2} \\
c^{2} d^{2} e^{2} f \\
c^{2} d e^{4} \\
c d^{4} e f \\
c d^{3} e^{3} \\
d^{6} f \\
d^{5} e^{2} \\
a^{1} b^{4} e^{3} \\
b^{3} c d f^{3} \\
c e^{2} f^{2} \\
d^{2} e f^{2} \\
d e^{3} f \\
e^{5} \\
b^{2} c^{3} f^{3} \\
c^{2} d e f^{2} \\
c^{2} e^{3} f \\
c d^{3} f^{2} \\
c d^{2} e^{2} f \\
c d e^{4} \\
d^{4} e f \\
d^{3} e^{3} \\
b^{1} c^{4} e f^{3} \\
c^{3} d^{2} f^{2} \\
c^{3} d e^{2} f\end{array}$ & 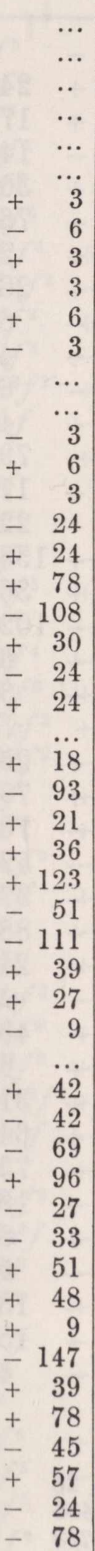 & $\begin{array}{rr}- & 9 \\
+ & 9 \\
+ & 45 \\
+ & 18 \\
- & 63 \\
- & 243 \\
+ & 351 \\
+ & 234 \\
- & 144 \\
- & 810 \\
+ & 900 \\
- & 288 \\
- & 36 \\
- & 9 \\
- & 144 \\
+ & 18 \\
+ & 243 \\
- & 1836 \\
+ & 3231 \\
+ & 3825 \\
- & 10620 \\
+ & 3888 \\
+ & 324 \\
+ & 1836 \\
- & 756 \\
+ & 1458 \\
- & 7938 \\
+ & 864 \\
+ & 2484 \\
+ & 16812 \\
- & 7488 \\
- & 15228 \\
+ & 7128 \\
+ & 3888 \\
- & 1944 \\
+ & 216 \\
+ & 2592 \\
- & 4293 \\
- & 4032 \\
+ & 8586 \\
- & 3645 \\
- & 2268 \\
+ & 9360 \\
+ & 1215 \\
- & 6624 \\
- & 6651 \\
+ & 4050 \\
+ & 4968 \\
- & 2970 \\
+ & 1296 \\
+ & 4428 \\
\end{array}$ & $\begin{array}{c}a^{1} b^{1} c^{3} e^{4} \\
c^{2} d^{3} e f \\
c^{2} d^{2} e^{3} \\
c d^{5} f \\
c d^{4} e^{2} \\
d^{6} e \\
b^{0} c^{5} d f^{2} \\
c^{5} e^{2} f \\
c^{4} d^{2} e f \\
c^{4} d e^{3} \\
c^{3} d^{4} f \\
c^{3} d^{3} e^{2} \\
c^{2} d^{5} e \\
c d^{7} \\
a^{0} b^{5} d f^{3} \\
e^{2} f^{2} \\
b^{4} c^{2} f^{3} \\
c d e f \\
c e^{3} f \\
d^{3} f^{2} \\
d^{2} e^{2} f \\
d e^{4} \\
b^{3} c^{3} e f^{2} \\
c^{2} d^{2} f^{2} \\
c^{2} d e^{2} f \\
c^{2} e^{4} \\
c d^{3} e f \\
c d^{2} e^{3} \\
d^{5} f \\
d^{4} e^{2} \\
b^{2} c^{4} d f^{2} \\
c^{4} e^{2} f \\
c^{3} d^{2} e f \\
c^{3} d e^{3} \\
c^{2} d^{4} f \\
c^{2} d^{3} e^{2} \\
c d^{5} e \\
d^{7} \\
b^{1} c^{6} f^{2} \\
c^{5} d e f \\
c^{5} e^{3} \\
c^{4} d^{3} f \\
c^{4} d^{3} e^{2} \\
c^{3} d^{4} e \\
c^{2} d^{6} \\
b^{0} c^{7} e f \\
c^{6} d^{2} f \\
c^{6} d e^{9} \\
c^{5} d^{3} e \\
c^{4} d^{5}\end{array}$ & $\begin{array}{lr}- & 60 \\
+ & 36 \\
+ & 108 \\
- & 24 \\
- & 6 \\
- & 9 \\
- & 9 \\
- & 51 \\
+ & 96 \\
+ & 111 \\
- & 27 \\
- & 234 \\
+ & 141 \\
- & 27 \\
- & 18 \\
+ & 18 \\
+ & 15 \\
+ & 33 \\
- & 63 \\
+ & 54 \\
- & 66 \\
+ & 27 \\
- & 54 \\
- & 129 \\
+ & 186 \\
+ & 45 \\
+ & 54 \\
- & 96 \\
- & 54 \\
+ & 48 \\
+ & 114 \\
+ & 9 \\
- & 150 \\
- & 147 \\
+ & 93 \\
+ & 150 \\
- & 87 \\
+ & 18 \\
- & 27 \\
- & 30 \\
+ & 30 \\
- & 6 \\
+ & 108 \\
- & 96 \\
+ & 21 \\
+ & 27 \\
- & 9 \\
- & 57 \\
+ & 51 \\
-\quad 12\end{array}$ & 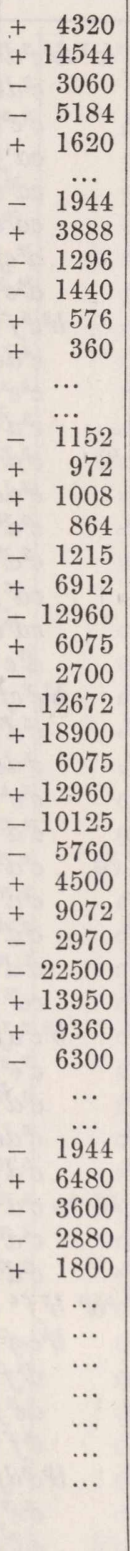 & $\begin{array}{c}a^{4} b^{e} e f^{4} \\
a^{3} b^{1} d f^{4} \\
e^{2} f^{3} \\
b^{0} c^{2} f^{4} \\
c d e f^{3} \\
c e^{3} f^{2} \\
d^{3} f^{3} \\
d^{2} e^{2} f^{2} \\
d e^{4} f \\
e^{6} \\
a^{2} b^{2} c f^{4} \\
d e f^{3} \\
e^{3} f^{2} \\
b^{1} c^{2} e f^{3} \\
c d^{2} f^{3} \\
c d e^{2} f^{2} \\
c e^{4} f \\
d^{3} e f^{2} \\
d^{2} e^{3} f \\
d e^{5} \\
b^{0} c^{3} d f^{3} \\
c^{3} e^{2} f^{2} \\
c^{2} d^{2} e f^{2} \\
c^{2} d e^{3} f \\
c^{2} e^{5} \\
c d^{4} f^{2} \\
c d^{3} e^{2} f \\
c d^{2} e^{4} \\
d^{5} e f \\
d^{4} e^{3} \\
a^{1} b^{4} f^{4} \\
b^{3} c e f^{3} \\
d^{2} f^{3} \\
d e^{2} f^{2} \\
e^{4} f \\
b^{2} c^{2} f^{3} \\
c^{2} e^{2} f^{2} \\
c d^{2} e f^{2} \\
c d e^{3} f \\
c e^{5} \\
d^{4} f^{2} \\
d^{3} e^{2} f \\
d^{2} e^{4} \\
b^{1} c^{4} f^{3} \\
c^{3} d e f^{2} \\
c^{3} e^{3} f \\
c^{2} d^{3} f^{2} \\
c^{2} d^{2} e^{2} f \\
c^{2} d e^{4} \\
c d^{4} e f \\
c d^{3} e^{3} \\
d^{6} f \\
\end{array}$ & $\begin{array}{lr}- & 15 \\
+ & 30 \\
- & 15 \\
+ & 15 \\
- & 30 \\
+ & 15 \\
+ & 9 \\
- & 9 \\
- & 21 \\
+ & 15 \\
+ & 6 \\
+ & 3 \\
+ & 21 \\
- & 24 \\
- & 9 \\
+ & 9 \\
& 6 \\
& 3 \\
+ & 9 \\
- & 18 \\
+ & 9 \\
+ & 6 \\
-\quad 6 \\
+\quad 6 \\
-\quad 24 \\
+\quad 18 \\
& 45 \\
+ & 96 \\
- & 51 \\
- & 9 \\
- & 30 \\
+ & 66 \\
+ & 84 \\
- & 36 \\
- & 102 \\
- & 174 \\
+ & 210 \\
+ & 63\end{array}$ & $\begin{array}{rr} & \\
- & 9 \\
+ & 9 \\
- & 21 \\
+ & 162 \\
- & 120 \\
+ & 81 \\
- & 486 \\
+ & 576 \\
- & 192 \\
+ & 78 \\
- & 99 \\
+ & 21 \\
- & 309 \\
- & 1026 \\
+ & 2160 \\
- & 672 \\
+ & 1863 \\
- & 3456 \\
+ & 1440 \\
+ & 738 \\
- & 120 \\
- & 2538 \\
+ & 864 \\
+ & 192 \\
+ & 324 \\
+ & 2592 \\
- & 1728 \\
- & 972 \\
+ & 648 \\
- & 48 \\
- & 12 \\
+ & 768 \\
- & 1023 \\
+ & 459 \\
+ & 564 \\
+ & 1053 \\
- & 2340 \\
- & 2094 \\
+ & 1080 \\
- & 3888 \\
+ & 9747 \\
- & 4410 \\
- & 756 \\
- & 2820 \\
- & 528 \\
+ & 8748 \\
+ & 8496 \\
- & 5280 \\
- & 24624 \\
+ & 14040 \\
+ & 7776\end{array}$ & $\begin{array}{c}a^{1} b^{1} d^{5} e^{2} \\
b^{0} c^{5} e f^{2} \\
c^{4} d^{2} f^{2} \\
c^{4} d e^{2} f \\
c^{4} e^{4} \\
c^{3} d^{3} e f \\
c^{3} d^{2} e^{3} \\
c^{2} d^{5} f \\
c^{2} d^{4} e^{2} \\
c d^{6} e \\
d^{8} \\
a^{0} b^{5} e f^{3} \\
b^{4} c d f^{3} \\
c e^{2} f^{2} \\
d^{2} e f^{2} \\
d^{3} e^{3} f \\
e^{5} \\
b^{3} c^{3} f^{3} \\
c^{2} d e f^{2} \\
c^{2} e^{3} f \\
c d^{3} f^{2} \\
c d^{2} e^{2} f \\
c d e^{4} \\
d^{4} e f \\
d^{3} e^{3} \\
b^{2} c^{4} f^{3} f^{2} \\
c^{3} d^{2} f^{2} \\
c^{3} 3 e^{2} f \\
c^{3} e^{4} \\
c^{2} d^{3} e f \\
c^{2} d^{2} e^{3} \\
c d^{5} f \\
c d^{4} e^{2} \\
d^{6} e \\
b^{1} c^{5} d f^{2} \\
c^{5} e^{2} f \\
c^{4} d^{2} e f \\
c^{4} d e^{3} \\
c^{3} d^{4} f \\
c^{3} d^{3} e^{2} \\
c^{2} d^{5} e \\
c d^{7} \\
b^{0} c^{7} f^{2} \\
c^{6} d e f \\
c^{6} e^{3} \\
c^{5} d^{3} f \\
c^{5} d^{2} e^{2} \\
c^{4} d^{5} e \\
c^{3} d^{5}\end{array}$ & $\begin{array}{lr}- & 72 \\
+ & 36 \\
- & 45 \\
- & 120 \\
- & 6 \\
+ & 204 \\
+ & 120 \\
- & 66 \\
- & 240 \\
+ & 144 \\
- & 27 \\
- & \quad 9 \\
-\quad 9 \\
+\quad 18 \\
+\quad 45 \\
-\quad 27 \\
+\quad 7 \\
+\quad 51 \\
-\quad 72 \\
+\quad 63 \\
-213 \\
+171 \\
+\quad 36 \\
-\quad 43 \\
-\quad 39 \\
-150 \\
+303 \\
-\quad 18 \\
+174 \\
-345 \\
-\quad 99 \\
+192 \\
-\quad 18 \\
+117 \\
-\quad 51 \\
-330 \\
+\quad 87 \\
+147 \\
+186 \\
-201 \\
+\quad 45 \\
-\quad 27 \\
+\quad 99 \\
+\quad 2 \\
-\quad 45 \\
-\quad 96 \\
+\quad 87 \\
-\quad 20 \\
\end{array}$ & 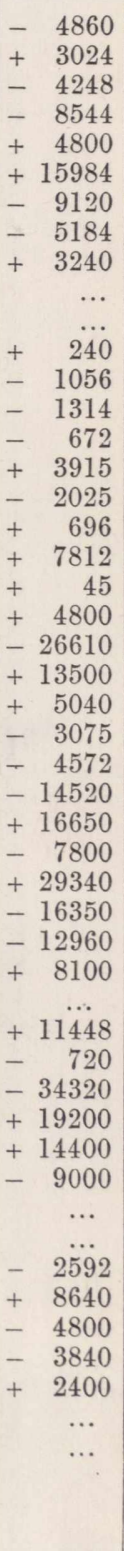 \\
\hline & & & & $\begin{array}{r} \pm \quad 12 \\
426 \\
912 \\
1101 \\
\pm 2451\end{array}$ & $\begin{array}{r} \pm \\
1548 \\
45999 \\
62019 \\
92853 \\
\pm 202428\end{array}$ & & & & & $\begin{array}{r} \pm \quad 8 \\
123 \\
1071 \\
1821 \\
\pm 3023\end{array}$ & $\begin{array}{r}\quad 828 \\
10920 \\
79779 \\
146226 \\
\pm 237753\end{array}$ \\
\hline
\end{tabular}


T. No. 94 .

$x$ coefficient.

$x$ coefficient.

\begin{tabular}{|c|c|c|c|c|c|c|c|}
\hline $\begin{array}{c}a^{5} b^{0} c f^{5} \\
d e f^{4} \\
e^{3} f^{3} \\
a^{4} b^{2} f^{5} \\
b^{1} c e f^{4} \\
d^{2} f^{4} \\
d e^{2} f^{3} \\
e^{4} f^{2} \\
b^{0} c^{2} d f^{4} \\
c^{2} e^{2} f^{3} \\
c d^{2} e f^{3} \\
c d e^{3} f^{2} \\
c e^{5} f^{4} \\
d^{4} f^{3} \\
d^{3} e^{2} f^{2} \\
d^{2} e^{4} f \\
d^{6} \\
a^{3} b^{3} e f^{4} \\
b^{2} c d f^{4} \\
c e^{2} f^{3} \\
d^{2} e f^{3} \\
d e^{3} f^{2} \\
e^{5} f \\
b^{1} c^{3} f^{4} \\
c^{2} d e f^{3} \\
c^{2} e^{3} f^{2} \\
c d^{3} f^{3} \\
c d^{2} e^{2} f^{2} \\
c d e^{4} f \\
c e^{6} \\
d^{4} e f^{2} \\
d^{3} e^{3} f \\
d^{2} e^{5} \\
b^{0} c^{4} e f^{3} \\
c^{3} d^{2} f^{3} \\
c^{3} d e^{2} f^{2} \\
c^{3} e^{4} f^{2} \\
c^{2} d^{3} e f^{2} \\
c^{2} d^{2} e^{3} f \\
c^{2} d e^{5} \\
c d^{5} f^{2} \\
c d^{4} e^{2} f^{2} \\
c d^{3} e^{4} \\
d^{6} e f \\
d^{5} e^{3} e^{3} \\
b^{4} d f^{4} \\
e^{2} f^{2} f^{3} \\
c d e f^{3} \\
c e^{3} f^{2} \\
\end{array}$ & 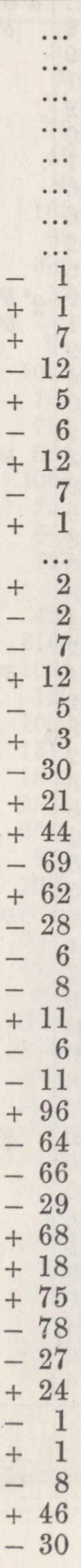 & 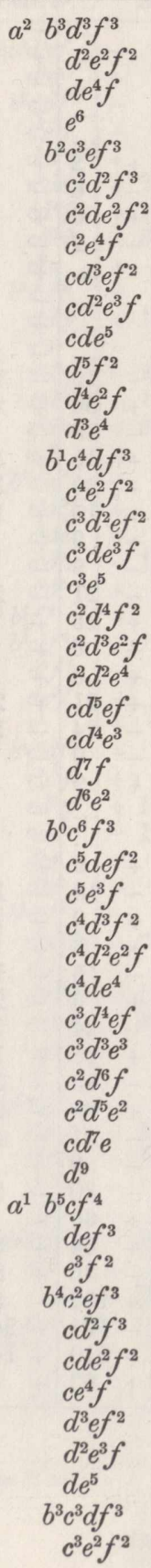 & 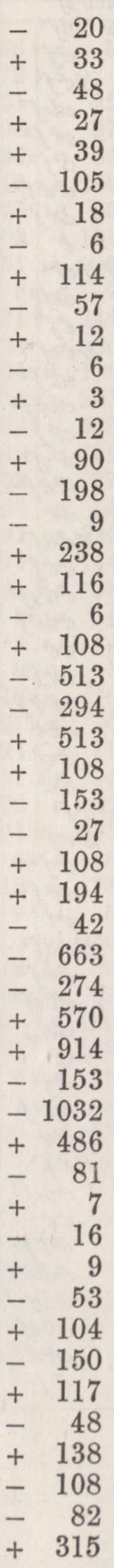 & $\begin{array}{c}a^{1} b^{3} c^{2} d^{2} e f^{2} \\
c^{2} d e^{3} f \\
c^{2} e^{5} \\
c d^{4} f^{2} \\
c d^{3} e^{2} f \\
c d^{2} e^{4} \\
d^{5} e f \\
d^{4} e^{3} \\
b^{2} c^{5} f^{3} \\
c^{4} d e f^{2} \\
c^{4} e^{3} f \\
c^{3} d^{3} f^{2} \\
c^{3} d^{2} e^{2} f \\
c^{3} d e^{4} \\
c^{2} d^{4} e f \\
c^{2} d^{3} e^{3} \\
c d^{6} f \\
c d^{5} e^{2} \\
d^{7} e \\
b^{1} c^{6} c f^{2} \\
c^{5} d^{2} f^{2} \\
c^{5} d e^{2} f \\
c^{5} e^{4} \\
c^{4} d^{3} e f \\
c^{4} d^{2} e^{3} \\
c^{3} d^{5} f \\
c^{3} d^{4} e^{2} \\
c^{2} d^{6} e \\
c d^{8} \\
b^{0} c^{7} d f^{2} \\
c^{7} e^{2} f \\
c^{6} d^{2} e f \\
c^{6} d e^{3} \\
c^{5} d^{4} f \\
c^{5} d^{3} e^{2} \\
c^{4} d^{5} e \\
c^{3} d^{7} \\
a^{0} c^{2} d f^{3} f^{3} \\
c^{2} e^{2} f^{2} \\
b^{6} d^{2} e e^{2} f^{3} \\
d^{5} f^{3} f \\
d^{4} f^{2} \\
d^{3} e^{2} f^{3} f \\
d^{2} e^{2} e^{4} \\
e^{4} f^{2} \\
b^{5}\end{array}$ & 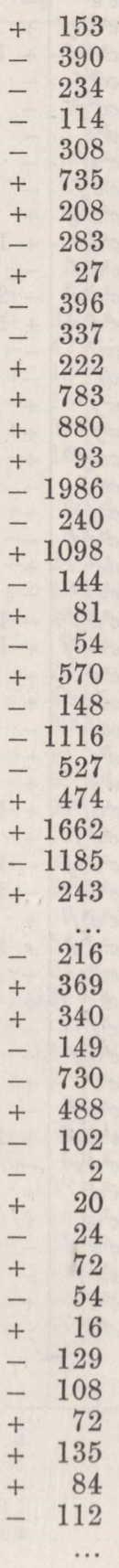 & $\begin{array}{c}a^{0} b^{4} c^{4} f^{3} \\
c^{3} d e f^{2} \\
c^{3} e^{3} f \\
c^{2} d^{3} f^{2} \\
c^{2} d^{2} e^{2} f \\
c^{2} d e^{4} \\
c d^{4} e f \\
c d^{3} e^{3} \\
d^{6} f \\
d^{5} e^{2} \\
b^{3} c^{5} e f^{2} \\
c^{4} d^{2} f^{2} \\
c^{4} d e^{2} f \\
c^{4} e^{4} \\
c^{3} d^{3} e f \\
c^{3} d^{2} e^{3} \\
c^{2} d^{5} f \\
c^{2} d^{4} e^{2} \\
c d^{6} e \\
d^{8} \\
b^{2} c^{6} d f^{2} \\
c^{6} e^{2} f \\
c^{5} d^{2} e f \\
c^{5} d e^{3} \\
c^{4} d^{4} f \\
c^{4} d^{3} e^{2} \\
c^{3} d^{5} e \\
c^{2} d^{7} \\
b^{1} c^{8} f^{2} \\
c^{7} d e f \\
c^{7} e^{3} \\
c^{6} d^{3} f \\
c^{6} d^{2} e^{2} \\
c^{5} d^{4} e \\
c^{4} d^{6} \\
b^{0} c^{9} e f \\
c^{8} d^{2} f \\
c^{8} d e^{2} \\
c^{7} d^{3} e \\
c^{6} d^{5}\end{array}$ & 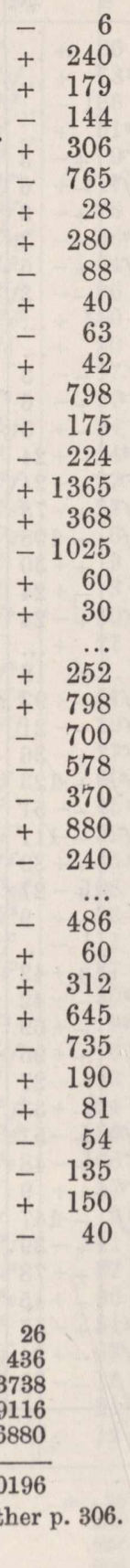 \\
\hline
\end{tabular}


T. No. 94 .

$y$ coefficient.

$y$ coefficient.

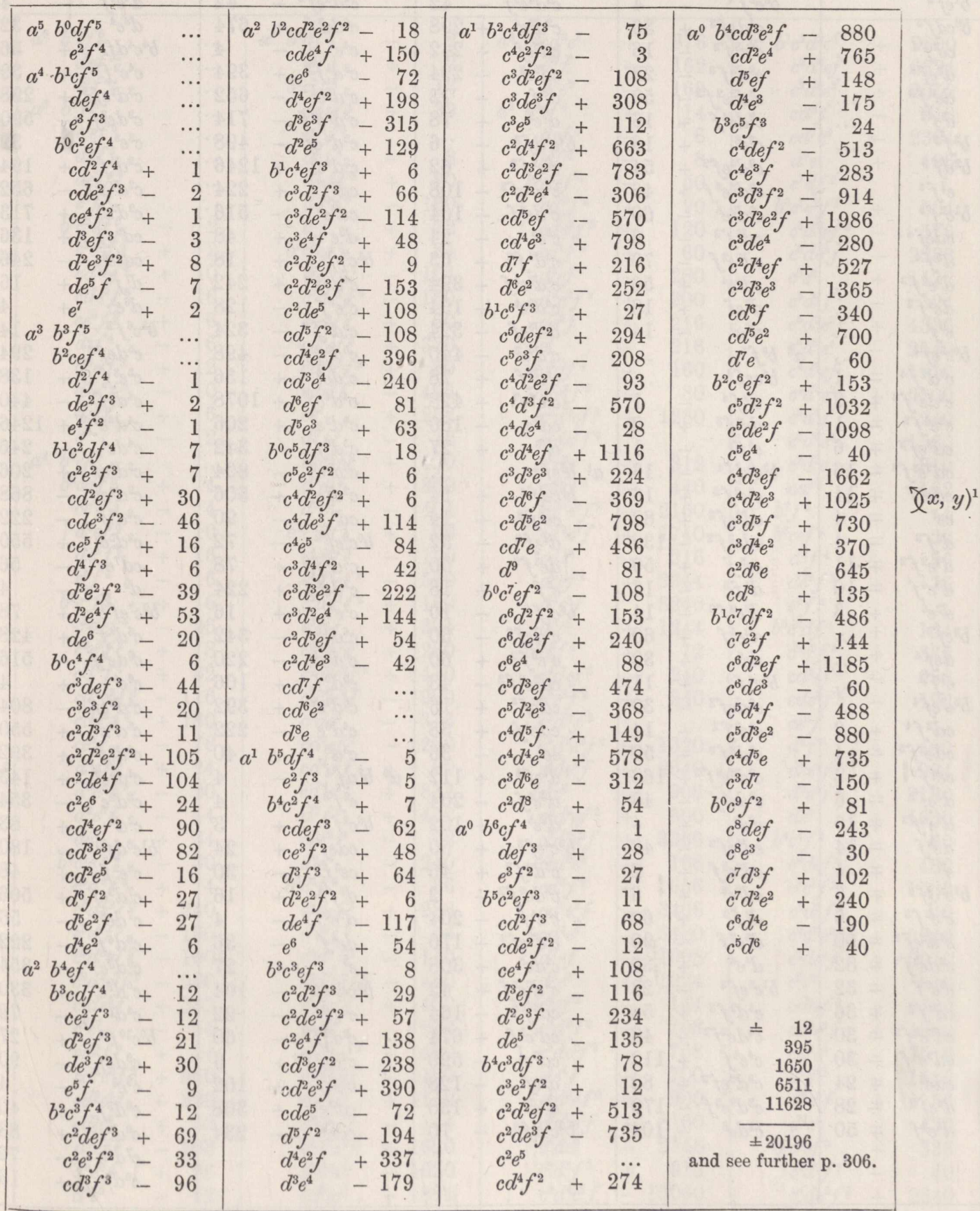


U. No. 29.

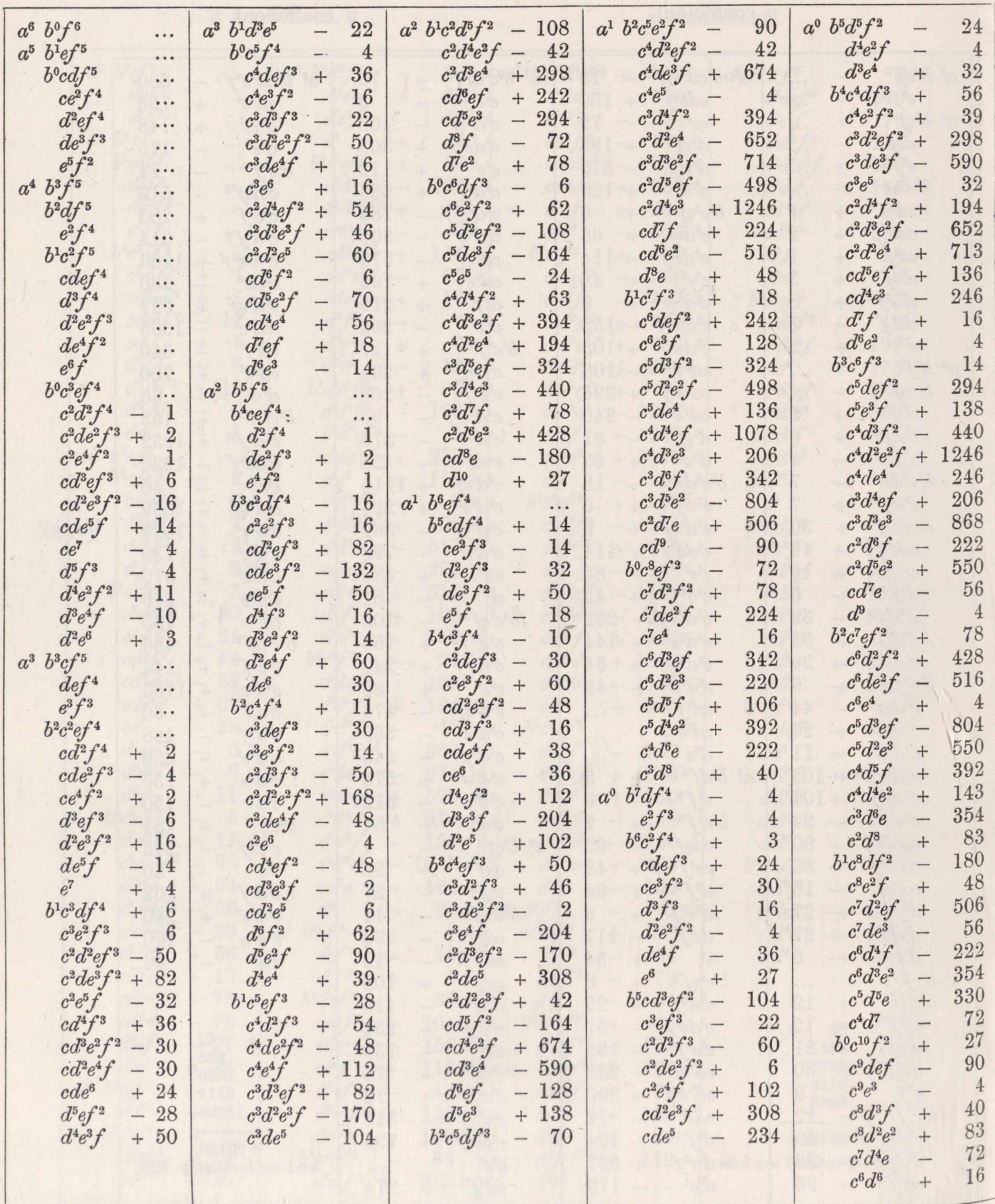

$\pm 36, \pm 464, \pm 2608, \pm 7278, \pm 6878$, together $\pm 17264:$ and see further $p .307$. 


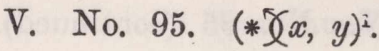

$x$ coefficient.

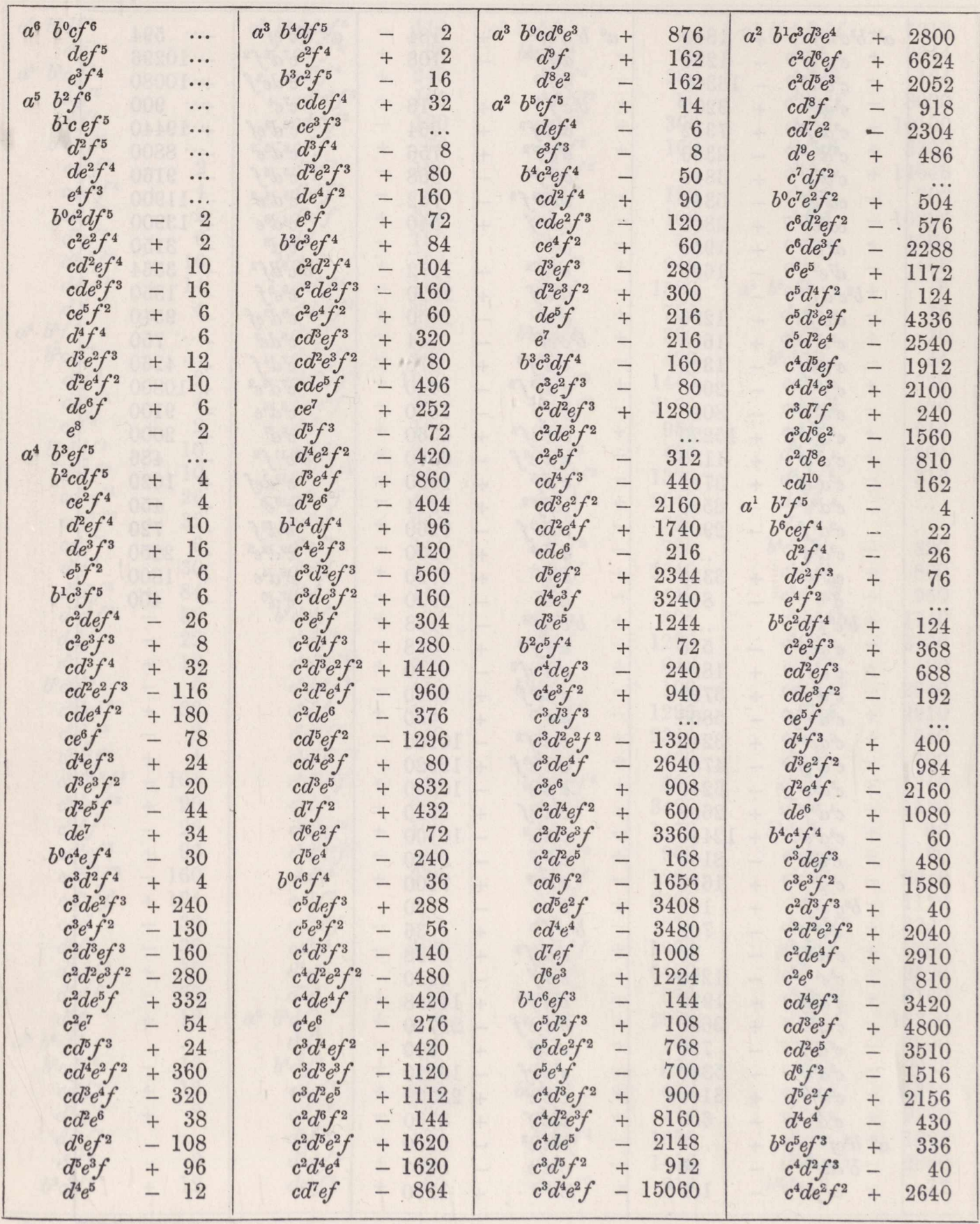

For the Numerical Verifications see p. 308. 
V. No. 95 (continued).

$x$ coefficient.

\begin{tabular}{|c|c|c|c|c|c|}
\hline $\begin{array}{c}a^{1} b^{3} c^{4} e^{4} f \\
c^{3} d^{3} e f^{2} \\
c^{3} d^{2} e^{3} f \\
c^{3} d e^{5} \\
c^{2} d^{5} f^{2} \\
c^{2} d^{4} e^{2} f \\
c^{2} d^{3} e^{4} \\
c d^{6} e f \\
c d^{5} e^{3} \\
d^{8} f \\
d^{7} e^{2} \\
b^{2} c^{6} d f^{3} \\
c^{6} e^{2} f^{2} \\
c^{5} d^{2} e f^{2} \\
c^{5} d e^{3} f \\
c^{5} e^{5} \\
c^{4} d^{4} f^{2} \\
c^{4} d^{3} e^{2} f \\
c^{4} d^{2} e^{4} \\
c^{3} d^{5} e f \\
c^{3} d^{4} e^{3} \\
c^{2} d^{7} f \\
c^{2} d^{6} e^{2} \\
c d^{8} e \\
d^{10} \\
b^{1} c^{8} f^{3} \\
c^{7} d e f^{2} \\
c^{7} e^{3} f \\
c^{6} d^{3} f^{2} \\
c^{6} d^{2} e^{2} f \\
c^{6} d e^{4} \\
c^{5} d^{4} e f \\
c^{5} d^{3} e^{3} \\
c^{4} d^{6} f \\
c^{4} d^{5} e^{2} \\
c^{3} d^{7} e \\
c^{2} d^{9} \\
b^{0} c^{9} e f^{2} \\
c^{8} d^{2} f^{2} \\
c^{8} d e^{2} f \\
c^{8} e^{4} \\
c^{7} d^{3} e f \\
c^{7} d^{2} e^{3} \\
c^{6} d^{5} f \\
c^{6} d^{4} d^{4} e^{2} \\
c^{5} d^{5} d^{6} f^{4} \\
c^{4} d^{2} f^{3} \\
\end{array}$ & 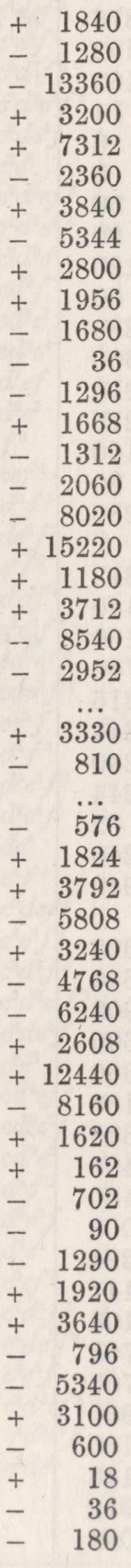 & $\begin{array}{c}a^{0} b^{7} d^{2} e f^{3} \\
d e^{3} f^{2} \\
e^{5} f \\
b^{6} c^{3} f^{4} \\
c^{2} d e f^{3} \\
c^{2} e^{3} f^{2} \\
c d^{3} f^{3} \\
c d^{2} e^{2} f^{2} \\
c d e^{4} f \\
c e^{6} \\
d^{4} e f^{2} \\
d^{3} e^{3} f \\
d^{2} e^{5} \\
b^{5} c^{4} f^{3} \\
c^{3} d^{2} f^{3} \\
c^{3} d e^{2} f^{2} \\
c^{3} e^{4} f^{5} \\
c^{2} d^{3} e f^{2} \\
c^{2} d^{2} e^{3} f \\
c^{2} d e^{5} \\
c d^{5} f^{2} \\
c d^{4} e^{2} f \\
c d^{3} e^{4} \\
d^{6} e f \\
d^{5} e^{3} \\
b^{4} c^{5} d f^{3} \\
c^{5} e^{2} f^{2} \\
c^{4} d^{2} e f^{2} \\
c^{4} d e^{3} f \\
c^{4} e^{5} \\
c^{3} d^{4} f^{2} \\
c^{3} d^{3} e^{2} f \\
c^{3} d^{2} e^{4} \\
c^{2} d^{5} e f \\
c^{2} d^{4} e^{3} \\
c d^{7} f \\
c d^{6} e^{2} \\
d^{8} e \\
b^{3} c^{7} f^{3} \\
c^{6} d e f^{2} \\
c^{6} e^{3} f \\
c^{5} d^{3} f^{2} \\
c^{5} d^{2} e^{2} f \\
c^{5} d e^{4} \\
c^{4} d^{4} e f \\
c^{4} d^{3} e^{3} \\
c^{3} d^{6} f \\
c^{3} d^{5} e^{2} \\
c^{2} d^{7} e \\
c d^{9} \\
c^{9}\end{array}$ & 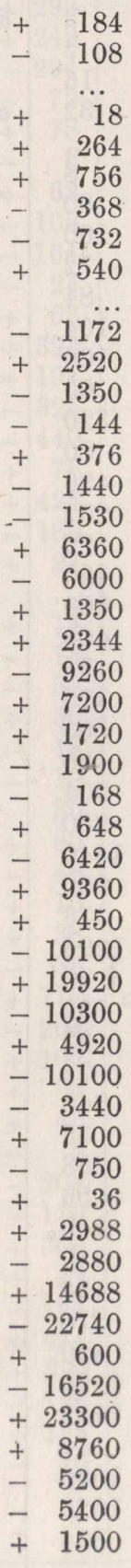 & $\begin{array}{c}a^{0} b^{2} c^{8} e f^{2} \\
c^{7} d^{2} f^{2} \\
c^{7} d e^{2} f \\
c^{7} e^{4} \\
c^{6} d^{3} e f \\
c^{6} d^{2} e^{3} \\
c^{5} d^{5} f \\
c^{5} d^{4} e^{2} \\
c^{4} d^{6} e \\
c^{3} d^{8} \\
b^{1} c^{9} d f^{2} \\
c^{9} e^{2} f \\
c^{8} d^{2} e f \\
c^{8} d e^{3} \\
c^{7} d^{4} f \\
c^{7} d^{3} e^{2} \\
c^{6} d^{5} e \\
c^{5} d^{7} \\
b^{0} c^{11} f^{2} \\
c^{10} d e f \\
c^{10} e^{3} \\
c^{9} d^{3} f \\
c^{9} d^{2} e^{2} \\
c^{8} d^{4} e \\
c^{7} d^{6}\end{array}$ & $\begin{array}{lr}- & 594 \\
- & 10296 \\
+ & 10080 \\
+ & 900 \\
+ & 19440 \\
- & 8800 \\
- & 9160 \\
+ & 11900 \\
+ & 13900 \\
- & 3150 \\
+ & 3564 \\
- & 1350 \\
- & 9540 \\
- & 750 \\
+ & 4260 \\
+ & 10800 \\
- & 9100 \\
+ & 2000 \\
- & 486 \\
+ & 1620 \\
+ & 450 \\
- & 720 \\
& 2250 \\
+ & 1800 \\
- & 400\end{array}$ \\
\hline
\end{tabular}


V. No. 95 (continued),

$y$ coefficient.



For the Numerical Verifications see p. 308.

C. II. 
V. No. 95 (concluded).

$y$ coefficient.

\begin{tabular}{|c|c|c|c|c|c|}
\hline $\begin{array}{c}a^{1} b^{2} c^{5} d^{2} f^{3} \\
c^{5} d e^{2} f^{2} \\
c^{5} e^{4} f^{4} \\
c^{4} d^{3} e f^{2} \\
c^{4} d^{2} e^{3} f \\
c^{4} d e^{5} \\
c^{3} d^{5} f^{2} \\
c^{3} d^{4} e^{2} f \\
c^{3} d^{3} e^{4} \\
c^{2} d^{6} e f \\
c^{2} d^{5} e^{3} \\
c d^{8} f \\
c d^{7} e^{2} \\
d^{9} e \\
b^{1} c^{7} d f^{3} \\
c^{7} e^{2} f^{2} \\
c^{6} d^{2} e f^{2} \\
c^{6} d e^{3} f \\
c^{6} e^{5} \\
c^{5} d^{4} f^{2} \\
c^{5} d^{3} e^{2} f \\
c^{5} d^{2} e^{4} \\
c^{4} d^{5} e f \\
c^{4} d^{4} e^{3} \\
c^{3} d^{3} f \\
c^{3} d^{6} e^{2} \\
c^{2} d^{8} e \\
c d^{10} \\
b^{0} c^{9} f^{3} \\
c^{8} d e f^{2} \\
c^{8} e^{3} f \\
c^{7} d^{3} f^{2} \\
c^{7} d^{2} e^{2} f \\
c^{7} d e^{4} \\
c^{6} d^{4} e f \\
c^{6} d^{3} e^{3} \\
c^{5} d^{6} f \\
c^{5} d^{5} e^{2} \\
c^{4} d^{7} e \\
c^{3} d^{9} \\
a^{0} \quad b^{8} f^{5} \\
b^{7} c e f^{5} \\
d^{2} f^{4} \\
d^{2} e^{2} f^{3} \\
e^{4} f^{2} \\
b^{6} c^{2} d f^{4} \\
c^{2} e^{2} f^{3} \\
c d^{2} e f^{3} \\
c d e^{3} f^{2} \\
c^{5} f^{5} f\end{array}$ & $\begin{array}{lr}+ & 1620 \\
+ & 3408 \\
+ & 2156 \\
- & 15060 \\
- & 2360 \\
- & 9260 \\
+ & 4336 \\
+ & 15220 \\
+ & 19920 \\
- & 5808 \\
- & 22740 \\
- & 90 \\
+ & 10080 \\
- & 1350 \\
- & 864 \\
- & 1008 \\
+ & 6624 \\
- & 5344 \\
+ & 1720 \\
- & 1912 \\
+ & 3712 \\
+ & 4920 \\
- & 4768 \\
- & 16520 \\
+ & 1920 \\
+ & 19440 \\
- & 9540 \\
+ & 1620 \\
+ & 162 \\
- & 918 \\
+ & 1956 \\
+ & 240 \\
- & 2952 \\
- & 3440 \\
+ & 2608 \\
+ & 8760 \\
- & 796 \\
- & 9160 \\
+ & 4260 \\
- & 720 \\
- & 2 \\
+ & 34 \\
- & 54 \\
+ & 252 \\
- & 216 \\
+ & 38 \\
- & 404 \\
- & 376 \\
- & 216 \\
+ & 1080\end{array}$ & $\begin{array}{c}a^{0} b^{6} d^{4} f^{3} \\
d^{3} e^{2} f^{2} \\
d^{2} e^{4} f \\
d^{6} \\
b^{5} c^{4} f^{4} \\
c^{3} d e f^{3} \\
c^{3} e^{3} f^{2} \\
c^{2} d^{3} f^{3} \\
c^{2} d^{2} e^{2} f^{2} \\
c^{2} d e^{4} f \\
c^{2} e^{6} \\
c d^{4} e f^{2} \\
c d^{3} e^{3} f \\
c d^{2} e^{5} \\
d^{6} f^{2} \\
d^{5} e^{2} f \\
d^{4} e^{4} \\
b^{4} c^{5} e f^{3} \\
c^{4} d^{2} f^{3} \\
c^{4} d e^{2} f^{2} \\
c^{4} e^{4} f^{4} \\
c^{3} d^{3} e f^{2} \\
c^{3} d^{2} e^{3} f \\
c^{3} d e^{5} \\
c^{2} d^{5} f^{2} \\
c^{2} d^{4} e^{2} f \\
c^{2} d^{3} e^{4} \\
c d^{6} e f \\
c d^{5} e^{3} \\
d^{8} f \\
d^{7} e^{2} \\
b^{3} c^{6} d f^{3} \\
c^{6} e^{2} f^{2} \\
c^{5} d^{2} e f^{2} \\
c^{5} d e^{3} f \\
c^{5} e^{5} \\
c^{4} d^{4} f^{2} \\
c^{4} d^{3} e^{2} f \\
c^{4} d^{2} e^{4} \\
c^{3} d^{5} e f \\
c^{3} d^{4} e^{3} \\
c^{2} d^{7} f \\
c^{2} d^{6} e^{2} \\
c d^{8} e^{3} \\
d^{10} \\
b^{2} c^{8} f^{3} \\
c^{7} d e f^{2} \\
c^{7} e^{3} f \\
c^{6} d^{3} f^{2} \\
c^{6} d^{2} e^{2} f\end{array}$ & $\begin{array}{rr}- & 276 \\
+ & 908 \\
- & 810 \\
& 72 \\
- & 12 \\
+ & 832 \\
+ & 1244 \\
+ & 1112 \\
- & 168 \\
- & 3510 \\
- & 1350 \\
- & 2148 \\
+ & 3200 \\
+ & 1350 \\
+ & 1172 \\
- & 2060 \\
+ & 450 \\
- & 240 \\
- & 1620 \\
- & 3480 \\
- & 430 \\
+ & 2800 \\
+ & 3840 \\
+ & 7200 \\
- & 2540 \\
+ & 1180 \\
- & 10300 \\
+ & 3240 \\
+ & 600 \\
- & 1290 \\
+ & 900 \\
+ & 876 \\
+ & 1224 \\
+ & 2052 \\
+ & 2800 \\
- & 1900 \\
+ & 2100 \\
- & 8540 \\
- & 10100 \\
- & 6240 \\
+ & 23300 \\
+ & 3640 \\
- & 8800 \\
- & 750 \\
+ & 450 \\
- & 162 \\
- & 2304 \\
- & 1680 \\
- & 1560 \\
& \quad .\end{array}$ & $\begin{array}{c}a^{0} b^{2} c^{6} d e^{4} \\
c^{5} d^{4} e f \\
c^{5} d^{3} e^{3} \\
c^{4} d^{6} f \\
c^{4} d^{5} e^{2} \\
c^{3} d^{7} e \\
c^{2} d^{9} \\
b^{1} c^{9} e f^{2} \\
c^{8} d^{2} f^{2} \\
c^{8} d e^{2} f \\
c^{8} e^{4} \\
c^{7} d^{3} e f \\
c^{7} d^{2} e^{3} \\
c^{6} d^{5} f \\
c^{6} d^{4} e^{2} \\
c^{5} d^{6} e \\
c^{4} d^{8} \\
b^{0} c^{10} d f^{2} \\
c^{10} e^{2} f \\
c^{9} d^{2} e f \\
c^{9} d e^{3} \\
c^{8} d^{4} f \\
c^{8} d^{3} e^{2} \\
c^{7} d^{5} e \\
c^{6} d^{7}\end{array}$ & $\begin{array}{lr}+ & 7100 \\
+ & 12440 \\
- & 5200 \\
- & 5340 \\
- & 11900 \\
+ & 10800 \\
- & 2250 \\
+ & 486 \\
+ & 810 \\
+ & 3330 \\
- & 750 \\
- & 8160 \\
- & 5400 \\
+ & 3100 \\
+ & 13900 \\
- & 9100 \\
+ & 1800 \\
- & 162 \\
- & 810 \\
+ & 1620 \\
+ & 1500 \\
- & 600 \\
- & 3150 \\
+ & 2000 \\
- & 400\end{array}$ \\
\hline
\end{tabular}


W, $29 \mathrm{~A}$.

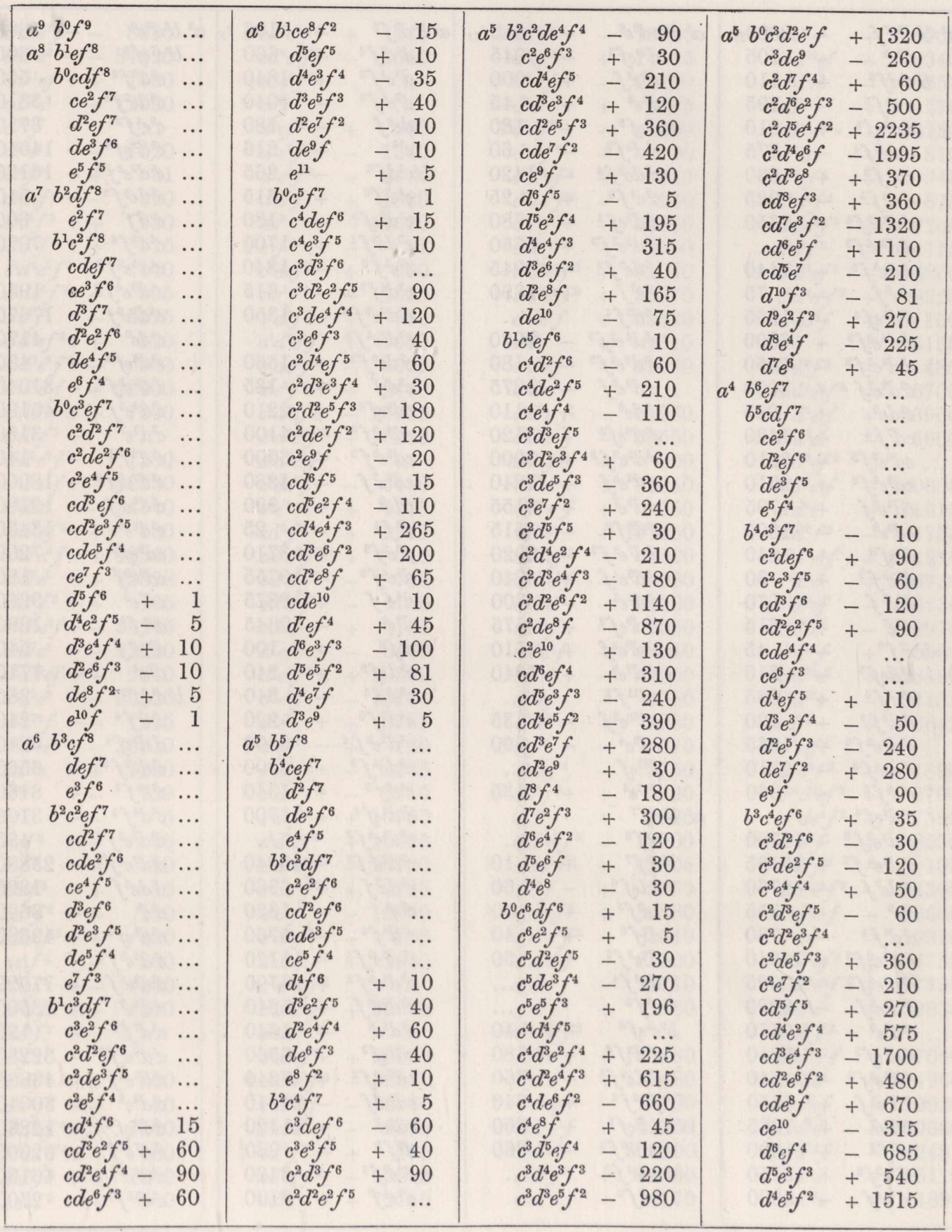

For the Numerical Verifications see p. 309. 
W, $29 \mathrm{~A}$ (continued).

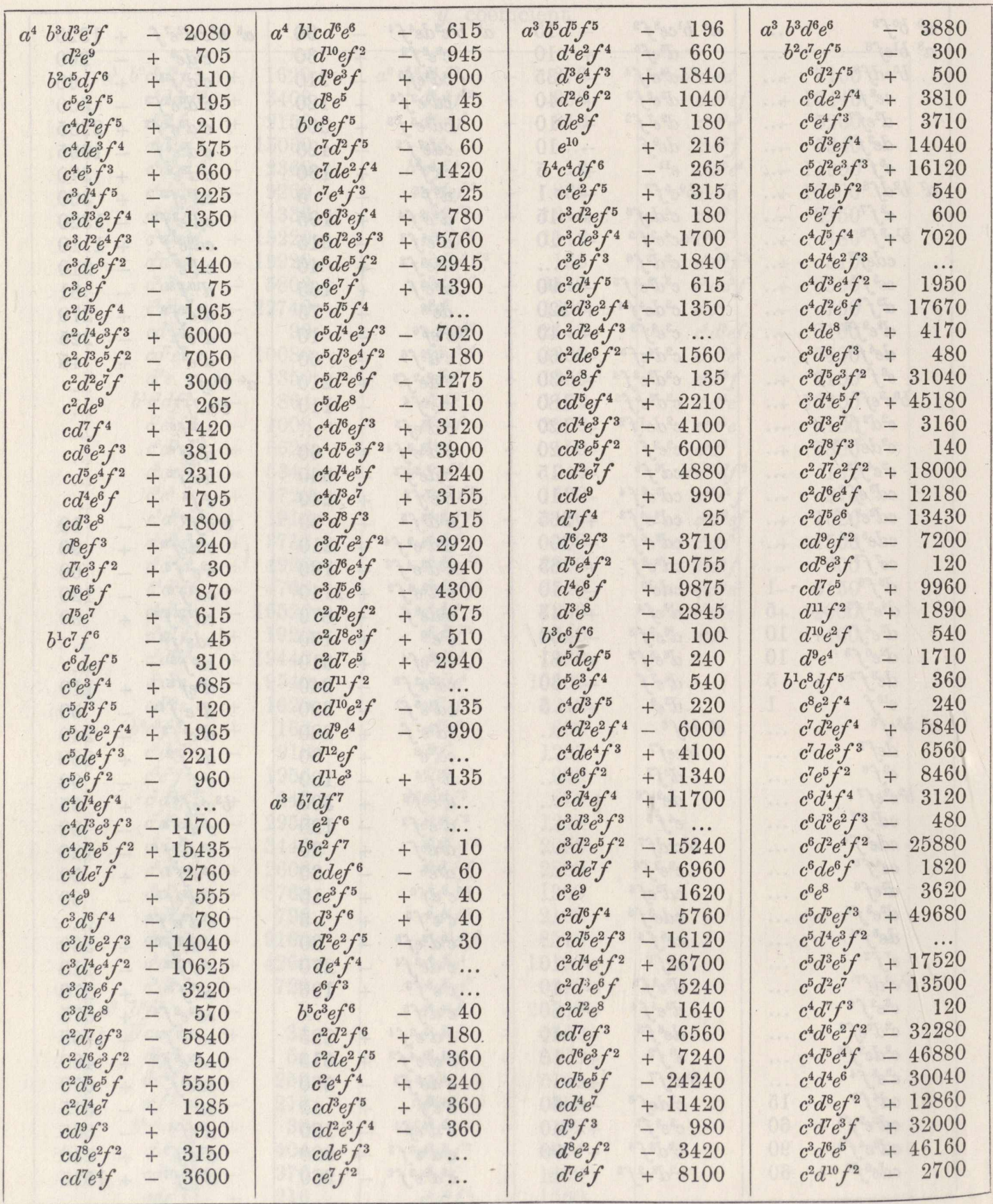


W, 29 A (continued).

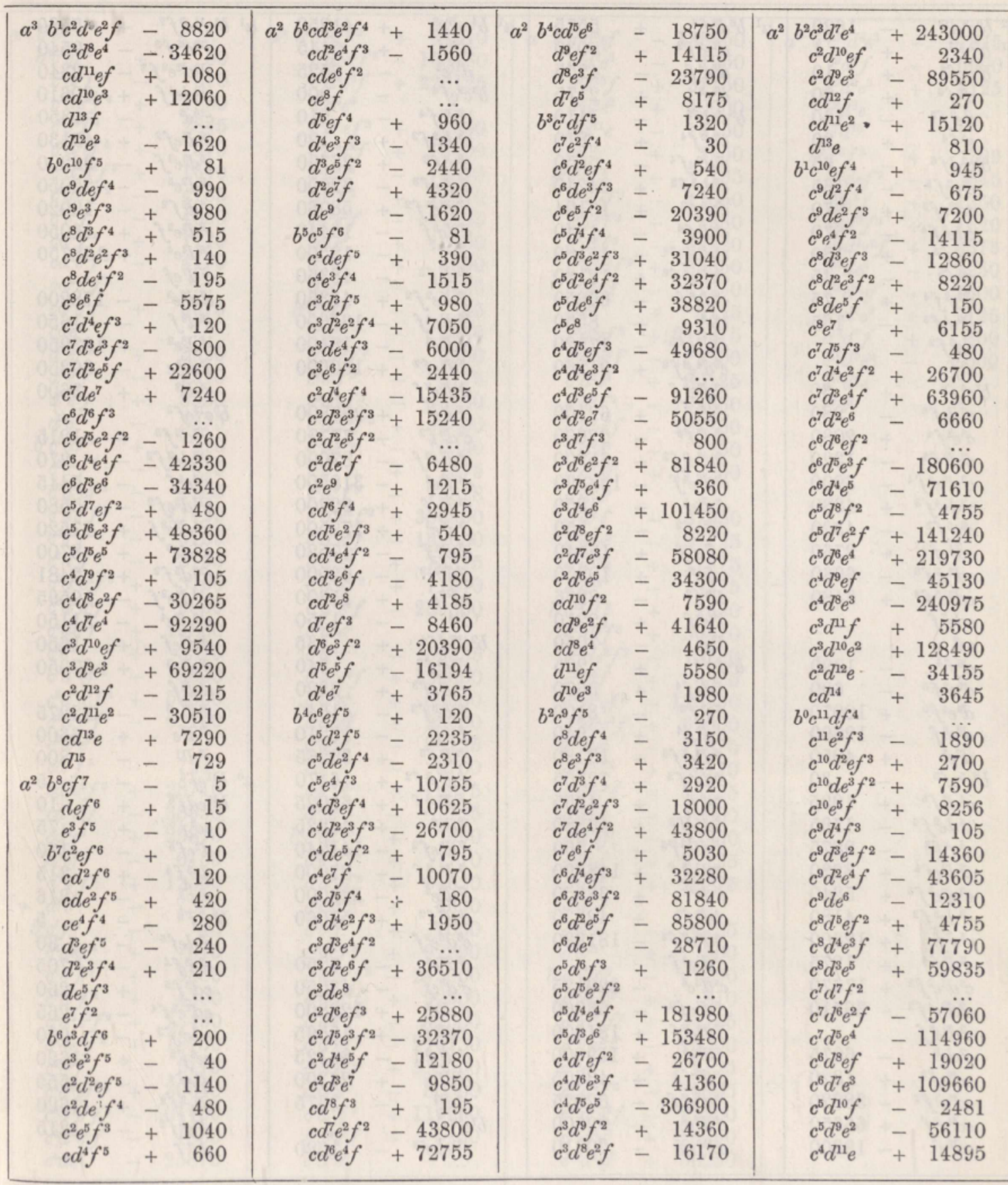


W, 29 A (continued).

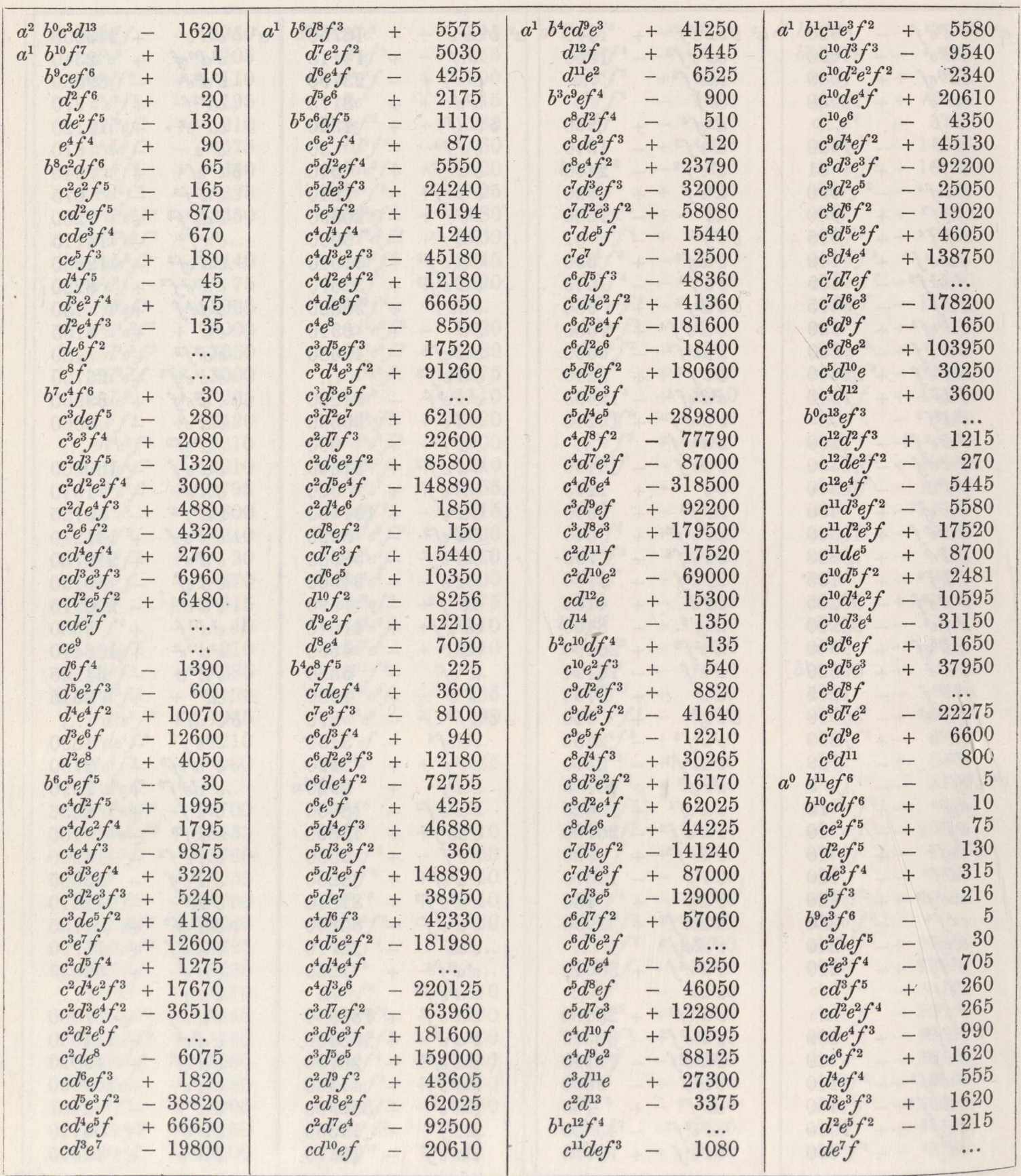


W, 29 A (concluded).

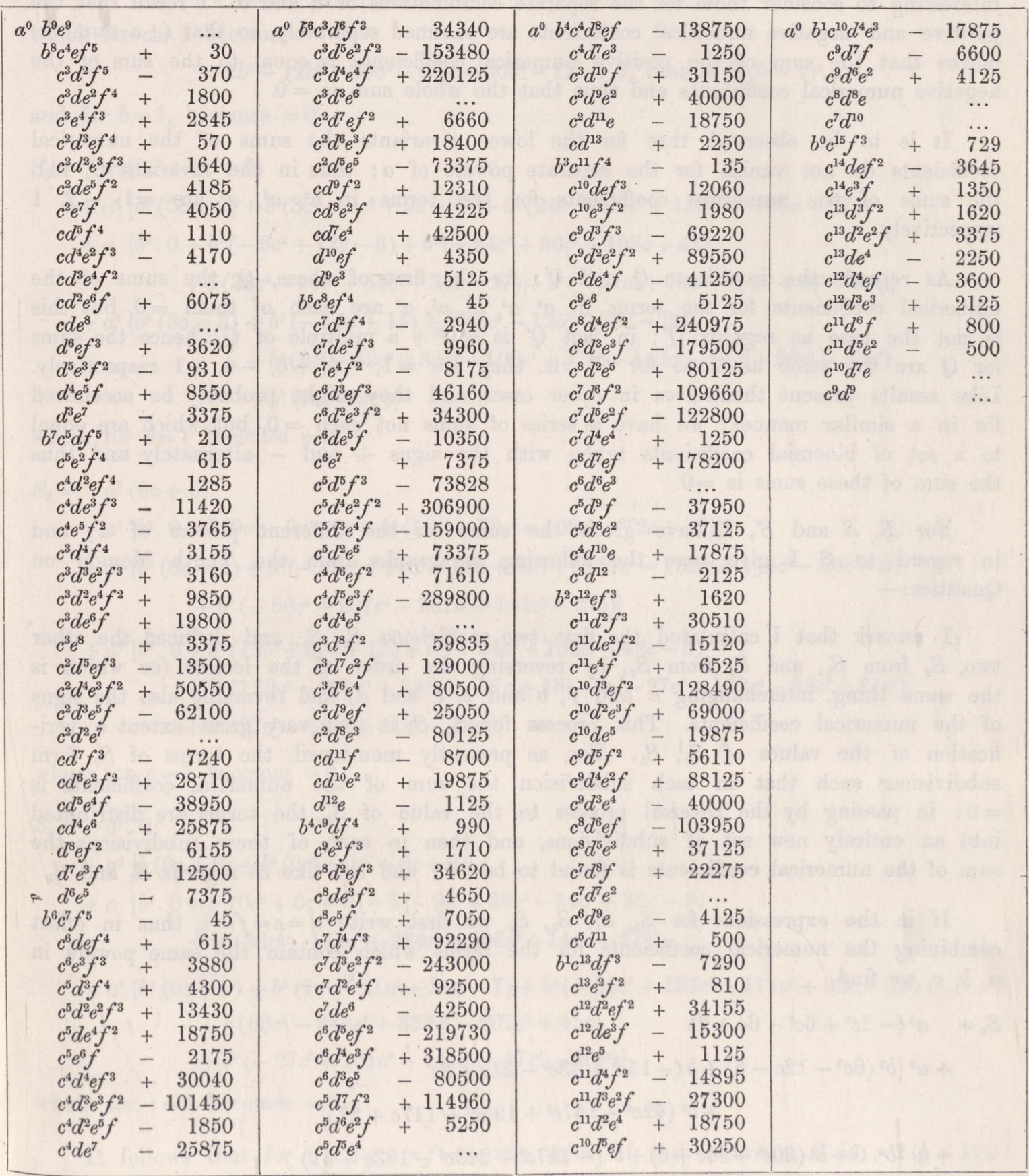


For the lower covariants the numerical verifications are given for the entire coefficient, but for the higher ones where the number of terms in a coefficient is considerable they are given separately for the different powers of $a$; and it is also interesting to consider them for the separate combinations of $a$ and $b$. I recall that the positive and negative numerical coefficients are summed separately, so that ( \pm a number) means that the sum of the positive numerical coefficients is equal to the sum of the negative numerical coefficients and thus that the whole sum is $=0$.

It is to be observed that for the lower covariants the sums of the numerical coefficients do not vanish for the separate powers of $a$ : thus in the invariant $G, 141$, the sums of the numerical coefficients for the terms in $a^{2}, a^{1}, a^{0}$ are $=1,-2,1$ respectively.

As regards the invariants $Q$ and $Q^{\prime}$; for the first of these, $Q$, the sums of the numerical coefficients for the terms in $a^{4}, a^{3}, a^{2}, a^{1}, a^{0}$ are each of them $=0$, but this is not the case as regards $Q^{\prime}$; in fact $Q^{\prime}$ is $=G^{2}+$ a multiple of $Q$; hence the sums for $Q$ are the same as those for $G^{2}$, viz. they are $=1,-4,+6,-4,+1$ respectively. Like results present themselves in other cases, and they might probably be accounted for in a similar manner; we have a series of sums not each $=0$, but which are equal to a set of binomial coefficients taken with the signs + and - alternately and thus the sum of these sums is $=0$.

For $R, S$ and $S^{\prime}$, I have given the sums for the different powers of $a$; and in regard to $S$ I give here the following paragraphs from the Tenth Memoir on Quantics:-

I remark that I calculated the first two coefficients $S_{0}, S_{1}$, and deduced the other two, $S_{2}$ from $S_{1}$, and $S_{3}$ from $S_{0}$, by reversing the order of the letters (or which is the same thing, interchanging $a$ and $f, b$ and $e, c$ and $d$ ) and reversing also the signs of the numerical coefficients. This process for $S_{2}, S_{3}$ is to a very great extent a verification of the values of $S_{0}, S_{1}$. For, as presently mentioned, the terms of $S_{0}$ form subdivisions such that in each subdivision the sum of the numerical coefficients is $=0$ : in passing by the reversal process to the value of $S_{3}$, the terms are distributed into an entirely new set of subdivisions, and then in each of these subdivisions the sum of the numerical coefficients is found to be $=0$; and the like as regards $S_{1}$ and $S_{3}$.

If in the expressions for $S_{0}, S_{1}, S_{2}, S_{3}$ we first write $d=e=f=1$, thus in effect combining the numerical coefficients for the terms which contain the same powers in $a, b, c$, we find

$$
\begin{aligned}
S_{0}= & a^{3}\left(-2 c^{3}+6 c^{2}-6 c+2\right) \\
+ & a^{2}\left\{b^{2}\left(6 c^{2}-12 c-6\right)+b\left(-15 c^{3}+33 c^{2}-21 c+3\right)\right. \\
& \left.+b^{0}\left(42 c^{4}-147 c^{3}+195 c^{2}-117 c+27\right)\right\} \\
+ & a\left\{b^{4} .0+b^{3}\left(30 c^{2}-36 c+6\right)+b^{2}\left(-117 c^{3}+249 c^{2}-183 c+51\right)\right. \\
& \left.+b\left(9 c^{5}+148 c^{4}-378 c^{3}+330 c^{2}-99 c\right)+b^{0}\left(-63 c^{6}+165 c^{5}-147 c^{4}+45 c^{3}\right)\right\}
\end{aligned}
$$




$$
\begin{aligned}
+a^{0} .\left\{b^{6} .2\right. & +b^{5}(-15 c+3)+b^{4}\left(75 c^{2}-69 c+24\right)+b^{3}\left(-9 c^{4}-167 c^{3}+225 c^{2}-87 c-2\right) \\
& +b^{2}\left(72 c^{5}+48 c^{4}-186 c^{3}+96 c^{2}\right)+b\left(-126 c^{6}+201 c^{5}-87 c^{4}\right) \\
& \left.+b^{0}\left(27 c^{8}-45 c^{7}+20 c^{6}\right)\right\}
\end{aligned}
$$

which for $c=1$ becomes

$$
=2 b^{6}-12 b^{5}+30 b^{4}-40 b^{3}+30 b^{2}-12 b+2, \text { that is } 2(b-1)^{6},
$$

and for $b=1$, becomes $=0$.

$$
\begin{aligned}
S_{2}= & a^{3}\left(0 c^{2}+0 c+0\right) \\
+ & a^{2}\left\{b^{2}(0 c+0)+b\left(3 c^{3}-9 c^{2}+9 c-3\right)+b^{0}\left(24 c^{4}-99 c^{3}+153 c^{2}-105 c+27\right)\right\} \\
+ & a\left\{b^{4} .0+b^{3}\left(-6 c^{2}+12 c-6\right)+b^{2}\left(-24 c^{3}+90 c^{2}-108 c+42\right)\right. \\
& \left.+b\left(33 c^{4}-90 c^{3}+54 c^{2}+30 c-27\right)+b^{0}\left(-27 c^{6}+78 c^{5}-66 c^{4}+6 c^{3}+9 c^{2}\right)\right\} \\
+ & a^{0}\left\{b^{5}(3 c-3)+b^{4}(-15 c+15)+b^{3}\left(6 c^{3}-12 c^{2}+36 c-30\right)\right. \\
& +b^{2}\left(9 c^{5}-42 c^{4}+84 c^{3}-108 c^{2}+57 c\right)+b\left(9 c^{6}-54 c^{5}+96 c^{4}-51 c^{3}\right) \\
& \left.+b^{0}\left(9 c^{7}-9 c^{6}\right)\right\}
\end{aligned}
$$

which for $c=1$ becomes $=0$.

$$
\begin{aligned}
S_{3}= & a^{3}(0 c+0) \\
+ & a^{2}\left\{b^{2} .0+b\left(0 c^{2}+0 c+0\right)+b^{0}\left(18 c^{4}-72 c^{3}+108 c^{2}-72 c+18\right)\right\} \\
+ & a\left\{b^{3}(0 c+0)+b^{2}\left(-33 c^{3}+99 c^{2}-99 c+33\right)+b\left(57 c^{4}-162 c^{3}+144 c^{2}-30 c-9\right)\right. \\
& \left.+b^{0}\left(-60 c^{5}+207 c^{4}-261 c^{3}+141 c^{2}-27 c\right)\right\} \\
+ & a^{0}\left\{b^{5} .0+b^{4}\left(15 c^{2}-30 c+15\right)+b^{3}\left(-54 c^{3}+102 c^{2}-42 c-6\right)\right. \\
& +b^{2}\left(123 c^{4}-297 c^{3}+243 c^{2}-87 c+18\right)+b\left(-27 c^{6}+102 c^{4}-96 c^{3}+21 c^{2}\right) \\
& \left.+b^{0}\left(27 c^{7}-60 c^{6}+51 c^{5}-12 c^{4}\right)\right\}
\end{aligned}
$$

which for $c=1$ becomes $=0$.

$$
\begin{aligned}
S_{4}= & a^{3} \cdot 0 \\
+ & a^{2}\left\{b(0 c+0)+b^{0}\left(0 c^{3}+0 c^{2}+0 c+0\right)\right\} \\
+ & a\left\{b^{3} \cdot 0+b^{2}\left(0 c^{2}+0 c+0\right)+b\left(-9 c^{4}+36 c^{3}-54 c^{2}+36 c-9\right)\right. \\
& \left.+b^{0}\left(36 c^{5}-171 c^{4}+324 c^{3}-306 c^{2}+144 c-27\right)\right\} \\
+ & a^{0}\left\{b^{4}(0 c+0)+b^{3}\left(7 c^{3}-21 c^{2}+21 c-7\right)+b^{2}\left(-39 c^{4}+135 c^{3}-171 c^{2}+93 c-18\right)\right. \\
& +b\left(66 c^{5}-243 c^{4}+333 c^{3}-201 c^{2}+45 c\right) \\
& \left.+b^{0}\left(-27 c^{7}+101 c^{6}-141 c^{5}+87 c^{4}-20 c^{3}\right)\right\}
\end{aligned}
$$

which for $c=1$ becomes $=0$.

It follows that for $c=d=e=f=1$, the value of the covariant $S$ is $=2(b-1)^{6} x^{3}$, which might be easily verified.

C. II. 
For $T, U, V$ and $W$, I look at the sums for the different combinations of $a$ and $b$. Thus for $T$ we have

$x$ coefficient.

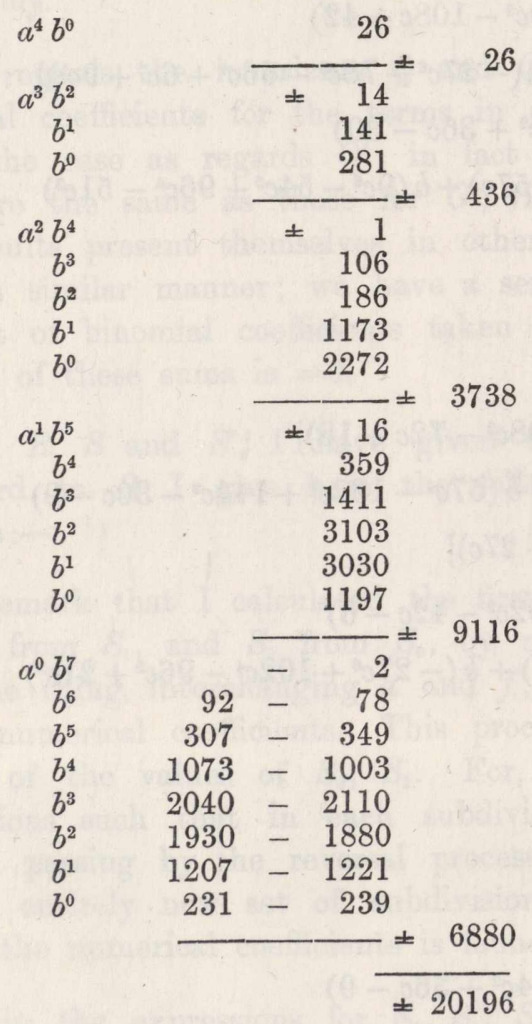

$y$ coefficient.

$a^{4} b^{0}$
$a^{3} b^{2}$
$b^{1}$
$b^{0}$
$a^{2} b^{3}$
$b^{2}$
$b^{1}$
$b^{0}$

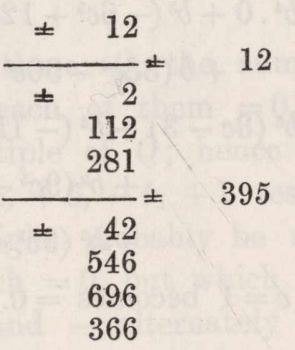

$a^{1} b^{5}$

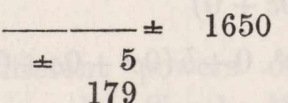

821

2097

2147

1262

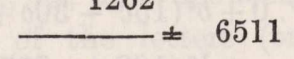

$a^{0} b^{6}$

$\pm \quad 28$

342

1790

3496

3445

2064

463

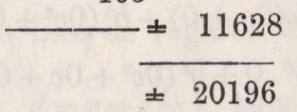

Observe here that in the $x$-coefficient for the terms in $a^{0}$ the successive sums are $-2,+14,-42,+70,-70,+42,-14+2$, which are the coefficients of $-2(\theta-1)^{7}$. 
For $U$ we have

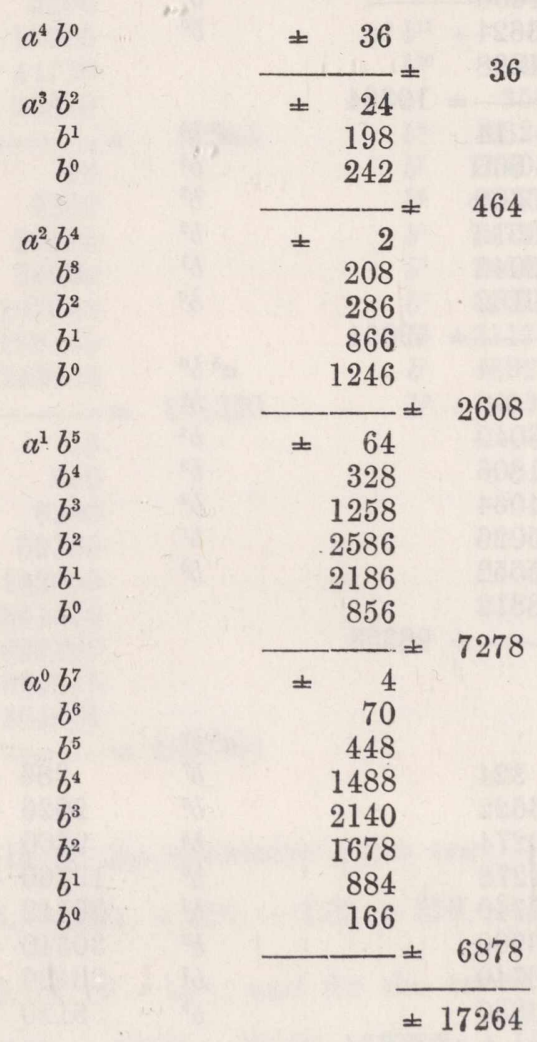


For $V$ we have

$$
x \text { coefficient. }
$$

$$
\begin{array}{r}
a^{5} b^{0} \\
a^{4} b^{2} \\
b^{1} \\
b^{0} \\
a^{3} b^{4}
\end{array}
$$

$b^{3}$

$b^{2}$

$b^{0}$

$a^{2} b^{5}$

$b^{4}$

$b^{3}$

$b^{2}$

$b^{1}$

$$
b^{0}
$$

$a^{1} b^{7}$

$\begin{array}{rrr}b^{7} & 4 \\ b^{6} & 76- & 48 \\ b^{5} & 2956- & 3040\end{array}$

b. $\quad 11946$ - 11806

$b^{3} \quad 23924-24064$

$b^{2} \quad 25110$ - 25026

$b^{1} \quad 25524-25552$

$b^{0} \quad 8822$ - 8812 $y$ coefficient.
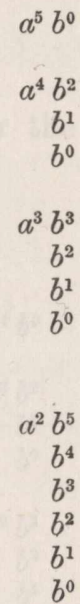

$a^{1} b^{6}$

$b^{5}$

$b^{4}$
$b^{3}$

$b^{2}$

$b^{1}$
$b^{0}$
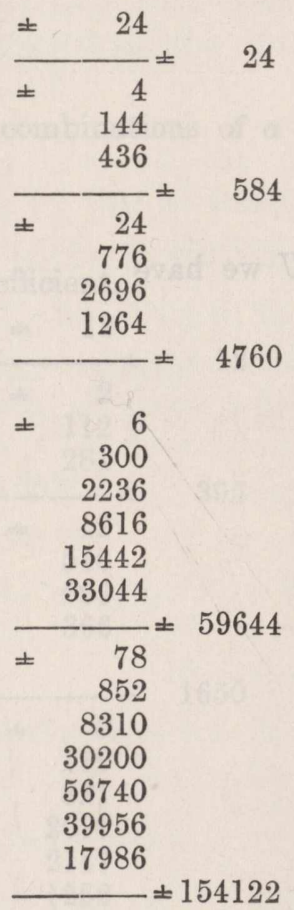

$\begin{array}{rrr}a^{0} b^{8} & 18 & \\ b^{7} & 184 & 324 \\ b^{6} & 4098- & 3622 \\ b^{5} & 19350- & 20274 \\ b^{4} & 42398- & 41278 \\ b^{3} & 51872-52740 \\ b^{2} & 44320- & 43900 \\ b^{1} & 20624- & 20740 \\ b^{0} & 3870- & 3856 \\ & & \pm 186734 \\ & & \pm 345894\end{array}$

Here in the $x$-coefficient for $a^{1}$ the successive sums are $-4,+28,-84,+140$, $-140,+84,-28,+4$, which are the coefficients of $-4(\theta-1)^{7}$; and for $a^{0}$ the successive sums are $18,-140,+476,-924,+1120,-868,+420,-116,+14$, which are the coefficients of $18(\theta-1)^{8}+4(\theta-1)^{7}$. In the $y$-coefficient the successive sums are $-2,+16,-56,+112,-140,+112,-56,+16,-2$, which are the coefficients of $-2(\theta-1)^{8}$. 
Finally for $W$ we have

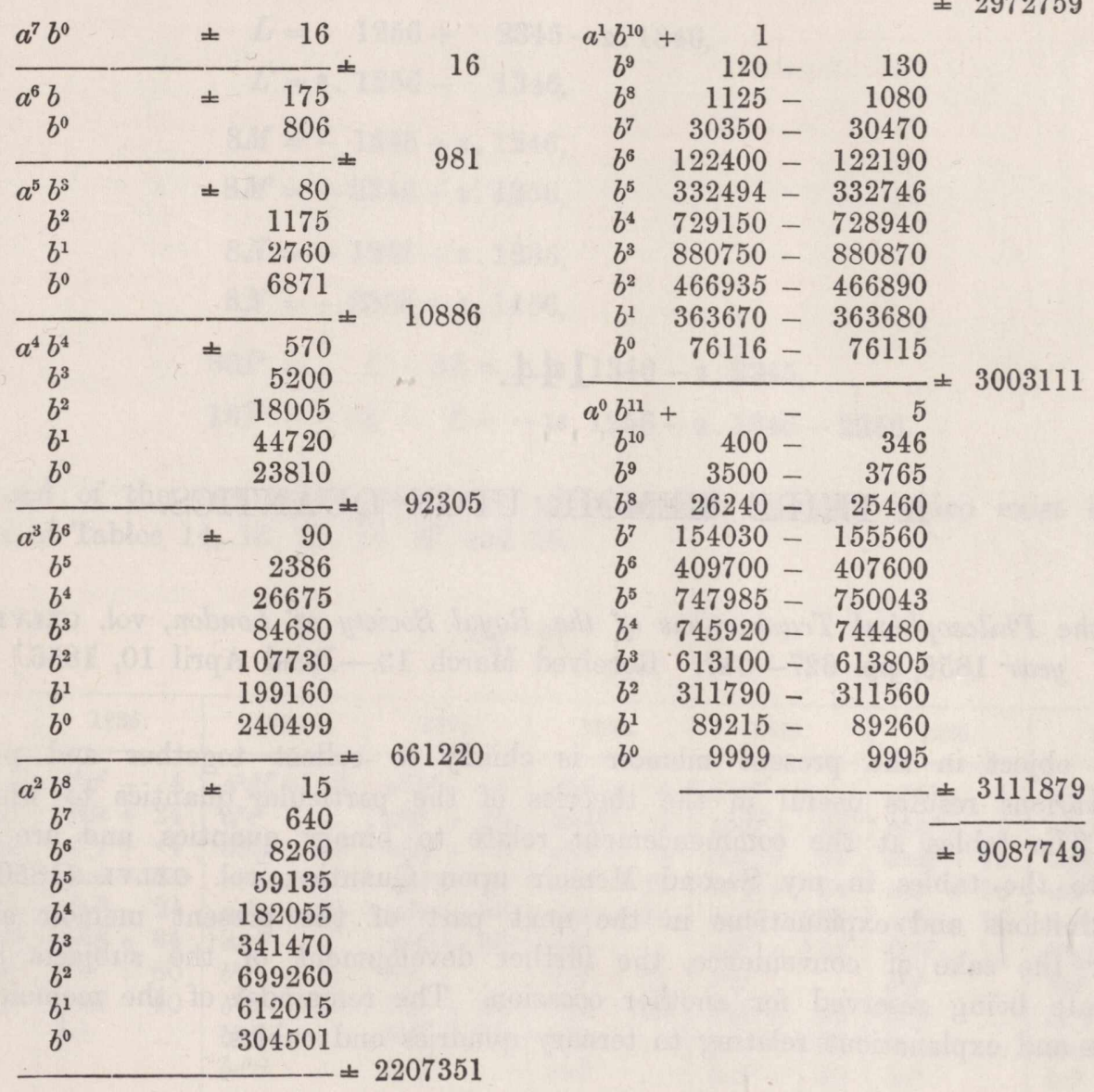

Here for the terms in $a^{1}$ the successive sums are

$$
1,-10,+45,-120,+210,-252,+210,-120,+45,-10,+1,
$$

which are the coefficients of $(\theta-1)^{10}$; and for the terms in $a^{0}$ the successive sums are

$$
-5,+54,-265,+780,-1530,+2100,-2058,+1440,-705,+230,-45,+4 \text {, }
$$

which are the coefficients of $-5(\theta-1)^{11}-(\theta-1)^{10}$. 